\title{
Comparative chloroplast genome analyses of Avena: insights into evolutionary dynamics and phylogeny
}

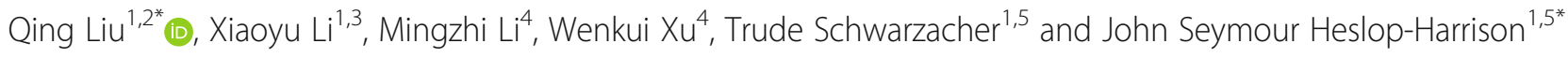

\begin{abstract}
Background: Oat (Avena sativa L.) is a recognized health-food, and the contributions of its different candidate Agenome progenitor species remain inconclusive. Here, we report chloroplast genome sequences of eleven Avena species, to examine the plastome evolutionary dynamics and analyze phylogenetic relationships between oat and its congeneric wild related species.
\end{abstract}

Results: The chloroplast genomes of eleven Avena species (size range of 135,889-135,998 bp) share quadripartite structure, comprising of a large single copy (LSC; 80,014-80,132 bp), a small single copy (SSC; 12,575-12,679 bp) and a pair of inverted repeats (IRs; 21,603-21,614 bp). The plastomes contain 131 genes including 84 protein-coding genes, eight ribosomal RNAs and 39 transfer RNAs. The nucleotide sequence diversities (Pi values) range from 0.0036 (rps 19) to 0.0093 (rp/32) for ten most polymorphic genes and from 0.0084 (psbH-petB) to 0.0240 (petG-trnWCCA) for ten most polymorphic intergenic regions. Gene selective pressure analysis shows that all protein-coding genes have been under purifying selection. The adjacent position relationships between tandem repeats, insertions/ deletions and single nucleotide polymorphisms support the evolutionary importance of tandem repeats in causing plastome mutations in Avena. Phylogenomic analyses, based on the complete plastome sequences and the LSC intermolecular recombination sequences, support the monophyly of Avena with two clades in the genus.

Conclusions: Diversification of Avena plastomes is explained by the presence of highly diverse genes and intergenic regions, LSC intermolecular recombination, and the co-occurrence of tandem repeat and indels or single nucleotide polymorphisms. The study demonstrates that the A-genome diploid-polyploid lineage maintains two subclades derived from different maternal ancestors, with A. longiglumis as the first diverging species in clade I. These genome resources will be helpful in elucidating the chloroplast genome structure, understanding the evolutionary dynamics at genus Avena and family Poaceae levels, and are potentially useful to exploit plastome variation in making hybrids for plant breeding.

Keywords: Avena, Chloroplast genome, Evolution rate, Insertions/deletions, Intermolecular recombination, Phylogenomics, Single nucleotide polymorphisms, Tandem repeats

\footnotetext{
* Correspondence: liuqing@scib.ac.cn; phh4@le.ac.uk

${ }^{1}$ Key Laboratory of Plant Resources Conservation and Sustainable Utilization /

Guangdong Provincial Key Laboratory of Applied Botany, South China

Botanical Garden, Chinese Academy of Sciences, Guangzhou, China

Full list of author information is available at the end of the article
}

(c) The Author(s). 2020 Open Access This article is licensed under a Creative Commons Attribution 4.0 International License, which permits use, sharing, adaptation, distribution and reproduction in any medium or format, as long as you give appropriate credit to the original author(s) and the source, provide a link to the Creative Commons licence, and indicate if changes were made. The images or other third party material in this article are included in the article's Creative Commons licence, unless indicated otherwise in a credit line to the material. If material is not included in the article's Creative Commons licence and your intended use is not permitted by statutory regulation or exceeds the permitted use, you will need to obtain permission directly from the copyright holder. To view a copy of this licence, visit http://creativecommons.org/licenses/by/4.0/. The Creative Commons Public Domain Dedication waiver (http://creativecommons.org/publicdomain/zero/1.0/) applies to the data made available in this article, unless otherwise stated in a credit line to the data. 


\section{Background}

Most chloroplast (cp) genomes (plastomes) of land plants have a typical quadripartite structure with a pair of inverted repeats (IRs) separated by a large single-copy (LSC) region and a small single-copy (SSC) region, and genome size ranging from the reduced plastome of 85 $\mathrm{kbp}$ in the non-photosynthetic gymnosperm Parasitaxus usta, up to $218 \mathrm{kbp}$ in Pelargonium [1, 2]. With high throughput sequencing methods [3], complete chloroplast genome sequences are widely used to improve phylogenetic resolution at the interspecific level and resolve the parentage of hybrid or polyploid taxa [4]. Some 866 plastid genomes of Poaceae have been assembled and deposited in the National Center for Biotechnology Information (NCBI:txid4479; accessed on 26 June 2020) Organelle Genome Resources since the publication of the first angiosperm chloroplast genome sequence of $\mathrm{Ni}$ cotiana tabacum [5]. The massive complete chloroplast genome sequences, together with knowledge about chloroplast sequence variation, means complete plastid sequencing has become an effective tool for plant phylogenomic analysis.

The plastome datasets have been widely used for resolving the recalcitrant phylogenetic relationships in plants, in part because the plastome has long been considered as a single evolutionary unit, meaning that genes can be concatenated in order to dissect phylogenetic signals $[6,7]$. Chloroplast genomes have also been identified with polymorphic regions caused by genomic expansions/contractions [8], inversions [9], gene rearrangements [10], etc. These mutational events in turn become synapomorphies for different plastid loci, sometimes yielding incongruent topologies. For example, three LSC inversions from trnS-GCU to $p s b A$ had been reported in some species of Poaceae [10], a LSC inversion from trnE-UUC to rpoB and a SSC inversion from rps15 to $n d h F$ were detected in some Asteraceae lineages [11, 12]. Whether such intermolecular recombinations exist in Avena plastomes remains uncertain, and their phylogenetic utilization merits further comparative study [13].

Oat (Avena sativa L., genomes AACCDD) is a recognized health food because of oat beta-glucan, which can actively reduce low-density lipoprotein cholesterol level and coronary heart disease risk [14]. The c. 29 species of Avena (Poaceae) are diverse in morphological and ecological terms, occurring across Asia, Europe and the Mediterranean Basin, Eastern Africa, and the Americas [15]. The genus includes diploids with A- or C-genomes $(2 x=14)$, and a polyploid series with $\mathrm{AB}$ - or AC-genome tetraploids $(4 x=28)$ and $A C D$-genome hexaploids $(6 x=$ 42) [16]. The A- and C-genome diploids are distinguished by the glume relative length and the chromosome structural differentiation [17], while the B and D genomes are not found in any extant diploids. The large size and highly repetitive nature of $A$. sativa genome has precluded the development of genomic breeding and selection of new oat varieties [18]. In recent phylogenetic analyses, oat was inferred to experience allopolyploidy events involving A-, C- and D-genome ancestors [16, 19], while the dynamic genomes with frequent chromosome translocations make it difficult to disentangle candidate progenitor species [20]. Plastid phylogenomics can be used for resolving contentious relationships $[6,7$, $11]$, so the plastome-inferred phylogenies between oat and its wild relatives deserve detailed evaluation.

Comparative plastome studies show evolution of tandem repeats, insertions/deletions (indels) and single nucleotide polymorphisms (SNPs), and the role of tandem repeats in generation of substitutions and indels [21, 22], with certain plastome regions being predisposed to indel and substitution mutations. If the distribution of plastome repeat sequences can be determined, it is feasible to predict microstructural variations by the correlation analyses between repeats, indels and substitutions. In addition to the paucity of genomic resources, the A-genome lineage phylogeny is enigmatic in Avena [19, 23, 24]. Thus, it is important to fully address polymorphic regions of Avena chloroplasts in an evolutionary context.

In current study, we report the chloroplast genome structure characterization, the polymorphic regions, and plastid phylogenomics in Avena using new plastid sequences and comparisons with published sequences. Our objectives were to: (1) gain insight into plastome structure features; (2) examine the intermolecular combination and microstructural variation domains; and (3) understand the evolutionary dynamics in selected species among 25 published Avena plastomes.

\section{Results}

\section{Plastome structure of Avena species}

Avena plastomes display a typical quadripartite circular structure containing one large single copy (LSC), one small single copy (SSC), and two inverted repeat (IRB and IRA) regions by GView [25] and OGDRAW [26] (Fig. 1, Additional files 1, 2: Tables S1, S2). Eleven Avena plastome size ranges from $135,889 \mathrm{bp}$ (A. brevis) to 135 , $998 \mathrm{bp}$ (A. wiestii). Average GC content is $38.48 \%$ (Table 1). Average coverage depth ranges from $1634 \times$ to $5339 \times$ (Table 1, Additional file 12: Figure S1).

Avena plastomes contain the same set of 131 genes encoding 84 proteins, eight ribosomal RNAs (rRNAs) and 39 transfer RNAs (tRNAs) (Table 2). In Avena plastomes, small fragments of truncated $n d h$ genes are detected in LSC ( $n d h C, 363 \mathrm{bp} ; n d h J, 480 \mathrm{bp}$ ) and SSC regions ( $n d h E, 306 \mathrm{bp}$ ) while only partial $n d h H$ in IRB region, the $\mathrm{C}$ (carboxy)-terminal part of $n d h H$ is encoded by the SSC block and its $\mathrm{N}$ (amino)-terminal 


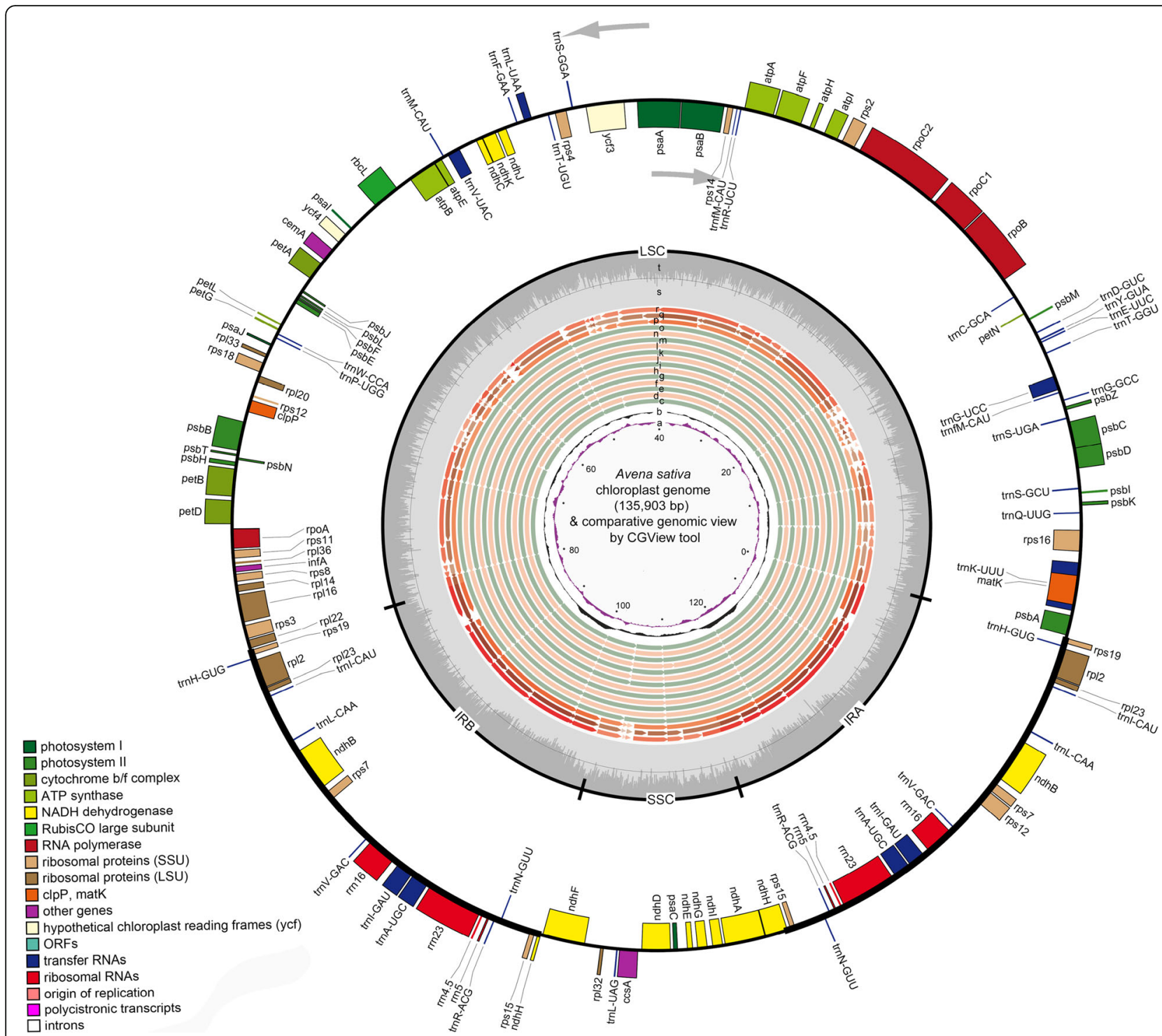

Fig. 1 Chloroplast genome map of Avena sativa_Liu312 (GWHAOPK01000000; the outer circle and rings "s-t") and GView comparison [25] of thirteen Avena species and three outgroup species plastomes (rings " $\mathrm{c}-\mathrm{r}^{\prime \prime}$ ). Genes belonging to different functional groups are shown in different colors. Genes shown on the outside of outer circle are transcribed counter-clockwise and on the inside clockwise. The tRNA genes are represented by one letter code of amino acids with anticodons. LSC, large single copy region; IR, inverted repeat; SSC, small single copy region. Rings " $a-b$ " from the innermost ring denote GC skews and GC content deviations from A. sativa_Liu312 plastome GC content, respectively; rings "C-r" denote the plastome sequence comparison by BLAST between $A$. sativa_Liu312 and other species plastomes outwards in turn: $A$. eriantha_Liu435, A. ventricosa_Liu275, A. atlantica_Liu437, A. wiestii_Liu439, A. strigosa_Liu 315, A. nuda_Liu443, A. hirtula_Liu299, A. sterilis_NC031650.1, A. sativa_NC027468.1, A. brevis_Liu289, A. sativa_Liu312, A. murphyi_Liu442, A. longiglumis_Liu438, wheat (Triticum aestivum_NC002762.1), maize (Zea mays_NC00166.1), and rice (Oryza sativa_NC008155.1); plastome similar and highly divergent locations are represented by continuous and interrupted track lines (except for 14 non-overlapping tracking bins across 16 rings "c-r"), respectively, with a heuristic considering the total number of plastome bases (135,887 bp to 135,998 bp) contributing to hits and their scores with 0-14 nonoverlapping $10 \mathrm{kbp}$ tracking bins. Rings "s-t" denote AT and GC content of Avena sativa_Liu312 plastome (GWHAOPK01000000) by OGDRAW [26]

part by the neighbouring IRB region. The remaining $n d h$ genes are the complete gene size.

In oat plastome, the rpoC1 intron is absent (Additional file 13: Figure S2). The predicted amino acid sequence is extremely hydrophilic and anionic, suggesting that the corresponding region may be post-translationally removed. Neither sequences nor amino acids encoded by
rpoC1 gene have been altered excessively by the intron loss (Additional file 14: Figure S3). ClpP had lost its two introns in Avena plastomes. Nineteen genes are duplicated in IRs from rps19 to rps15, including seven protein-coding genes (rps19, rpl2, rpl23, ndhB, rps7, rps12, and rps15), of which two use non-ATG start codons (rps19, GTG and rpl2, ACG; Table 2, Fig. 1). There are 16 different intron- 


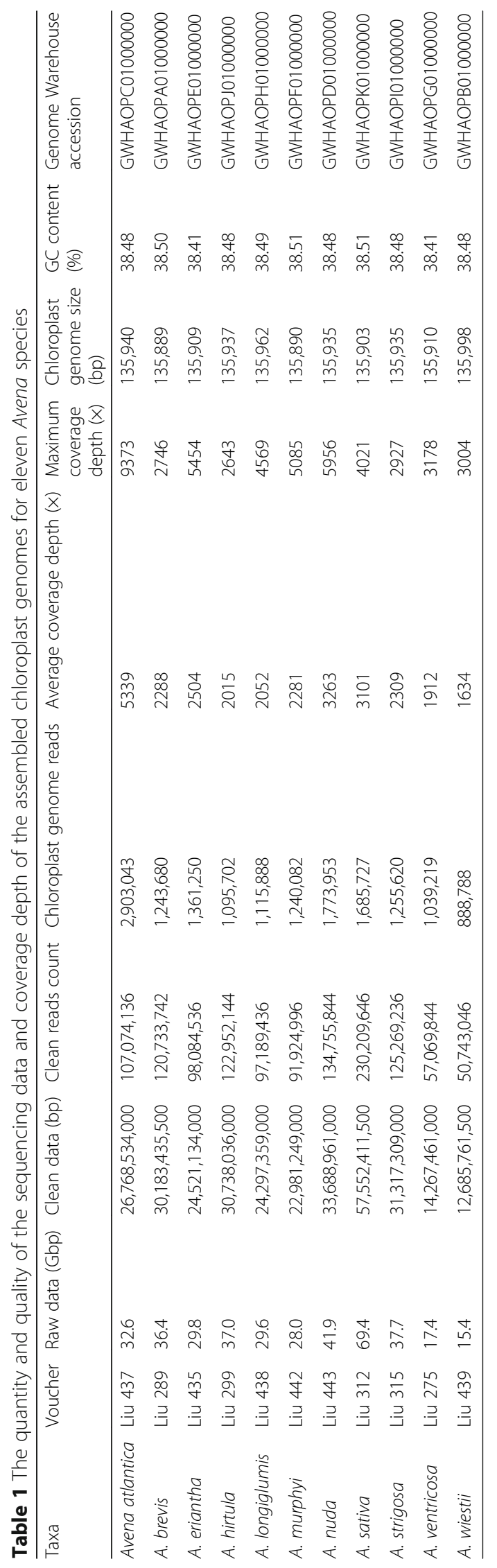


Table 2 Genes present in Avena plastomes

\begin{tabular}{|c|c|c|}
\hline Category of genes & Group of genes (gene number) & Gene name \\
\hline \multirow[t]{6}{*}{ Self replication } & Ribosomal RNAs (8) & rRNA16', rRNA23', rRNA4. $5^{c}, r R N A 5^{C}$ \\
\hline & Transfer RNAs (39) & 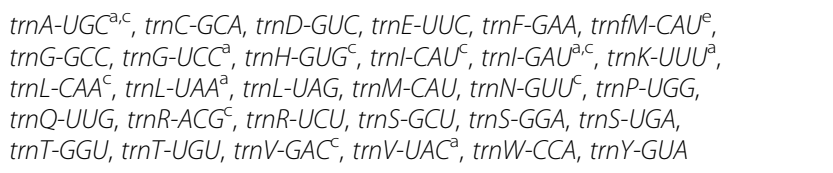 \\
\hline & Ribosomal protein (small subunit) (16) & $r p s 2, r p s 3, r p s 4, r p s 7^{c}, r p s 8, r p s 11, r p s 12^{a, d}, r p s 14, r p s 15^{c}, r p s 16^{a}, r p s 18, r p s 19^{c}$ \\
\hline & Ribosomal protein (large subunit) (11) & $r p / 2^{\mathrm{a}, \mathrm{c}}, r p / 14, r p / 16^{\mathrm{a}}, r p / 20, r p / 22, r p / 23^{\mathrm{c}}, r p / 32, r p / 33, r p / 36$ \\
\hline & DNA dependent RNA polymerase (4) & $r p o A, r p o B, r p o C 1, r p o C 2$ \\
\hline & Translation-related gene (1) & $\operatorname{infA}$ \\
\hline \multirow[t]{7}{*}{ Genes for photosynthesis } & Subunits of photosystem I (5) & psaA, psaB, psaC, psal, psaJ \\
\hline & Subunits of photosystem II (15) & $\begin{array}{l}p s b A, p s b B, p s b C, p s b D, p s b E, p s b F, p s b H, p s b l, p s b J, p s b K, \\
p s b L, p s b M, p s b N, p s b T, p s b Z\end{array}$ \\
\hline & Subunits of cytochrome b/f complex (6) & petA, petBa, petDa, petG, petL, petN \\
\hline & Subunits of ATP synthase (6) & $\operatorname{atp} A, \operatorname{atp} B, \operatorname{atp} E, \operatorname{atp} F^{a}, \operatorname{atpH}, a t p l$ \\
\hline & Subunits of NADH dehydrogenase (13) & $n d h A^{a}, n d h B^{a, c}, n d h C, n d h D, n d h E, n d h F, n d h G, n d h H^{c}, n d h l, n d h J, n d h K$ \\
\hline & ATP-dependent protease subunit (1) & $c l p P$ \\
\hline & Rubisco large subunit (1) & $r b c L$ \\
\hline \multirow[t]{3}{*}{ Other genes } & Maturase (1) & matk \\
\hline & Envelop membrane protein (1) & cemA \\
\hline & c-type cytochrome biogenesis (1) & $\operatorname{ccs} A$ \\
\hline Genes of unknown function & Conserved open reading frames (2) & $y c f 3^{b}, y c f 4$ \\
\hline
\end{tabular}

a Gene containing a single intron;

b Gene containing two introns;

c Two gene copies in the IRs;

d Gene divided into two independent transcription units;

e Duplicated gene in LSC region

containing genes, of which six are tRNA coding genes and rps12 and $y c f 3$ contain two introns. The trnK-UUU has the largest intron $(2435 \mathrm{bp})$ with $m a t K$ located within it (Table 3).

BLAST analyses of Avena species, wheat (Triticum aestivum_NC002762.1), maize (Zea mays_NC00166.1), and rice (Oryza sativa_NC008155.1) plastomes reflect the shared structural features. Avena plastomes share equivalent distribution patterns of GC islands where $\mathrm{G}$ and $C$ are distributed unevenly between DNA strands (rings "a-b" in Fig. 1). The lower overall sequence identity is shared by wheat, maize, and rice (rings "p-r" in Fig. 1) compared to the congeneric Avena species. In Avena, a high level of similarity is restricted to IRs, and major differences originate from LSC and SSC regions. Avena eriantha and $A$. ventricosa plastomes share five highly divergent sequence locations in LSC region (rps16-trnQ-UUG, trnC-GCA-rpoB, trnT-UGU-trnLUAA, ycf4-cemA, and psaJ-trnP-UGG) and one location in SSC region (ndhF-rpl32) to A. sativa_Liu312 plastome (rings "c-d" in Fig. 1). Avena atlantica, A. wiestii, A. strigosa, A. nuda, and A. hirtula share two highly divergent locations in LSC region (rps16-trnQ-UUG and $\operatorname{trn} V$ -
$U A C$-trnM-CAU) and one highly divergent location in SSC region (ndhF-rpl32) to A. sativa_Liu312 plastome (rings "e-i" in Fig. 1). Wheat, maize, and rice outwards in turn according to the phylogenetic location.

Avena sterilis, A. sativa, A. brevis, A. murphyi, and A. longiglumis plastomes show high similarity in LSC, SSC and IR regions (rings " $\mathrm{j}$-o" in Fig. 1), ribosomal gene clusters, tRNAs and the junction areas, where rpl22, rps19, $n d h H, n d h F$, and $p s b A$ are encoded (Additional file 15: Figure S4). Photosynthesis-related protein-coding genes in Avena plastomes show similarity to other angiosperms, including genes for ATP synthase, photosystem I and II, and RuBisCO. Other genes such as rps3 and $y c f 3$ show less conservation.

\section{Evolution between monocot and dicot plastomes: Avena and Taraxacum}

Mauve alignment [27] of plastomes show that the Avena plastome structure is similar to gramineous outgroups, Cenchrus, Hordeum, Oryza, Saccharum, Secale, Sorghum, Triticum, and Zea. At a higher resolution, two rearrangements are evident within LSC and IRB regions of oat, rice, wheat and Taraxacum amplum plastomes 
Table 3 Genes with intron(s) in Avena sativa plastome

\begin{tabular}{|c|c|c|c|c|c|c|}
\hline Gene & Region & Exon I (bp) & Intron I (bp) & Exon II (bp) & Intron II (bp) & Exon III (bp) \\
\hline $\operatorname{atpF}$ & LSC & $145^{+}$ & 825 & $407^{+}$ & & \\
\hline$n d h A$ & SSC & $550^{-}$ & 1021 & $539^{-}$ & & \\
\hline$n d h B$ & $\mathrm{IRB}$ & $777^{-}$ & 712 & $756^{-}$ & & \\
\hline$n d h B$ & IRA & $777^{+}$ & 712 & $756^{+}$ & & \\
\hline petB & LSC & $6^{+}$ & 759 & $642^{+}$ & & \\
\hline petD & LSC & $8^{+}$ & 741 & $475^{+}$ & & \\
\hline$r p / 2$ & $\mathrm{IRB}$ & $391^{-}$ & 663 & $431^{-}$ & & \\
\hline$r p / 2$ & IRA & $391^{+}$ & 663 & $431^{+}$ & & \\
\hline$r p / 16$ & LSC & $9^{-}$ & 1052 & $402^{-}$ & & \\
\hline $\operatorname{rps} 12^{\mathrm{a}}$ & $\mathrm{LSC}+\mathrm{IRB}$ & $114^{-}$ & - & $232^{-}$ & 799 & $29^{-}$ \\
\hline $\operatorname{rps} 12^{\mathrm{b}}$ & $\mathrm{LSC}+\mathrm{IRA}$ & $114^{-}$ & - & $232^{+}$ & 799 & $29^{+}$ \\
\hline rps16 & LSC & $40^{-}$ & 826 & $230^{-}$ & & \\
\hline $\operatorname{trn} A-U G C$ & IRB & $38^{+}$ & 811 & $35^{+}$ & & \\
\hline $\operatorname{trn} A-U G C$ & IRA & $38^{-}$ & 811 & $35^{-}$ & & \\
\hline $\operatorname{trnG-UCC}$ & LSC & $23^{-}$ & 677 & $48^{-}$ & & \\
\hline $\operatorname{trnl}-G A U$ & $\mathrm{IRB}$ & $37^{-}$ & 807 & $35^{-}$ & & \\
\hline $\operatorname{trnl}-G A U$ & IRA & $37^{+}$ & 807 & $35^{+}$ & & \\
\hline $\operatorname{trnK} K-U U U$ & LSC & $37^{-}$ & 2435 & $35^{-}$ & & \\
\hline $\operatorname{trn} L-U A A$ & LSC & $35^{+}$ & 33 & $50^{+}$ & & \\
\hline $\operatorname{trnV} V-U A C$ & $\mathrm{IRB}$ & $39^{+}$ & 596 & $37^{+}$ & & \\
\hline $\operatorname{trnV} V-U A C$ & IRA & $39^{-}$ & 596 & $37^{-}$ & & \\
\hline$y c f 3$ & LSC & $126^{-}$ & 755 & $226^{-}$ & $725^{-}$ & $161^{-}$ \\
\hline
\end{tabular}

Superscript ${ }^{+}$: exon is transcribed counter-clockwise in Fig. 1;

Superscript ${ }^{-}$: exon is transcribed clockwise in Fig. 1;

Hyphen-: spliceosomal intron;

a,b: The rps12 gene is divided into $5^{\prime}$-rps12 in LSC region, ${ }^{a} 3^{\prime}$-rps12 in IRB region and ${ }^{\text {b }} 3^{\prime}$-rps 12 in IRA region

(Fig. 2a). There is no interspecific and intraspecific rearrangements within eleven Avena species and two $A$. sativa accession plastomes (Fig. $2 \mathrm{~b}, \mathrm{c}$ ). A scheme is shown where the monocot and dicot structures could be derived from each other by two intermolecular recombination events (Fig. 3): (1) LSC recombination region starting from a dandelion-like ancestral gene order with starting point from trnC-GCA to trnfM at one end, an initial duplication of trnfM, further recombination between $\operatorname{trnC}$-GCA-trnR-UCU and psbD-trnfM (upstream), subsequently gave rise to the plastid DNA inversion of trnC-GCA-trnE-UUC occurred, encompassing about 31, $415 \mathrm{bp}$ sequence rearrangement of oat plastome (Fig. 3a); (2) IRB recombination region starting from a dandelion-like ancestral gene order with starting point from $y c f 1$ to $n d h F$ at one end, an initial loss of $y c f 1$, further duplication of $r p s 15-n d h H$, subsequently giving rise to the gene inversion of rps15-ndhF (downstream), encompassing about 13,758 bp sequence rearrangement of oat plastome (Fig. 3b).

The mVISTA [28] analysis shows overall sequence identity and divergent regions in Avena. A high degree of synteny and gene order conservation indicate an evolutionary conservation at plastome level (Fig. 4). Notably, LSC and SSC regions are more divergent than IRs, and non-coding regions show a higher sequence divergence than in coding regions (Additional file 3: Table S3). DnaSP [29] analysis shows nucleotide diversity of single copy genes and intergenic regions. Ten most polymorphic genes in descending order include rpl32, rpl16, psaC, psbF, ndhA, ndhC, atpF, matK, rpl22 and rps19, with the nucleotide diversity ( $\mathrm{Pi}$ ) values ranging from 0.0036 (rps19) to 0.0093 (rpl32) (Additional file 3: Table S3a, Fig. 5a). Among ten most polymorphic intergenic regions of petG-trnW-CCA, ccsA-ndhD, rpl16-rps3, trnRUCU-trnfM-CAU, rpl32-trnL-UAG, petB-petD, trnYGUA-trnD-GUC, ndhE-ndhG, rps8-rpl14 and psbH-petB, with the nucleotide diversity $(\mathrm{Pi})$ values ranging from 0.0084 (psbH-petB) to 0.0240 (petG-trnW-CCA) (Additional file 3: Table S3b, Fig. 5b). Six loci of LSC region and three loci of SSC region are identified for two datasets.

IR size is different among eleven Avena plastomes presented here available in Genome Warehouse database 


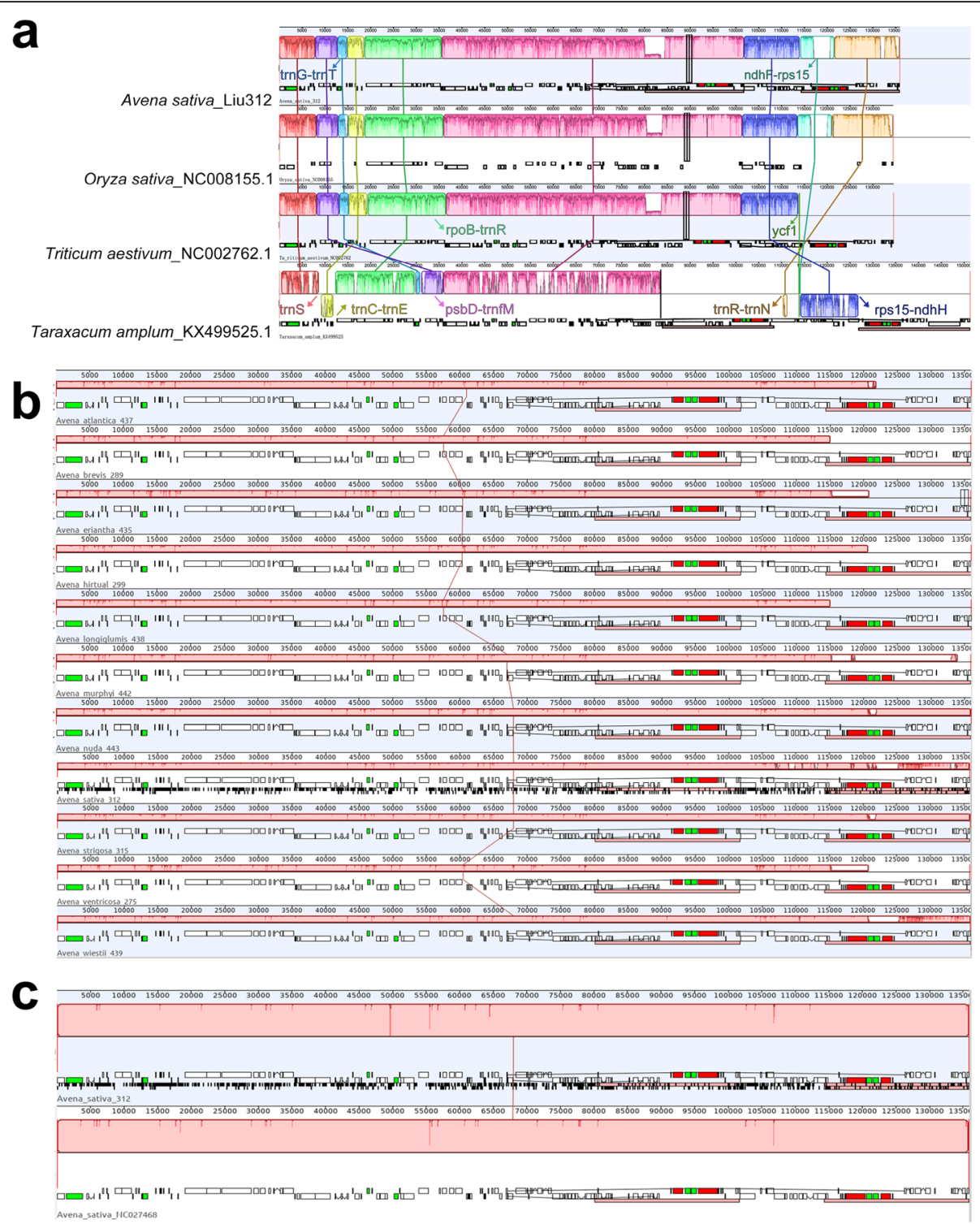

Fig. 2 Mauve alignment. a Oat, rice, wheat, and dandelion (Taraxacum amplum) plastomes from this study and NCBI revealed similarities and differences in syntenical blocks. Two rearrangements with respect to the dicot plastome with LSC and IRB intermolecular recombination please see Fig. 3. b Mauve alignment of eleven Avena plastomes revealing no interspecific rearrangement. c Mauve alignment of Avena sativa (GWHAOPK01000000 and NC027468.1) plastomes revealing no intraspecific rearrangement. Each colored block is a region of collinear sequence among investigated species plastomes. Blocks shown above and below the line are in opposite orientations

and fourteen species of different ploidies [representing a diploid maximum plastome size of $135,557 \mathrm{bp}$ of $A$. clauda to a hexaploid maximum plastome size of 135 , $900 \mathrm{bp}$ of $A$. hybrida among 25 Avena species] available in NCBI database (Additional file 15: Figure S4a, S4b), varying from $21,598 \mathrm{bp}$ in $A$. canariensis to $21,619 \mathrm{bp}$ in A. clauda. Avena plastomes have the same LSC/IRs and SSC/IRs borders, with two copies of rps $1936 \mathrm{bp}$ from LSC/IRs borders, with LSC rpl22 being 42 bp from LSC/ IRB border, and LSC psbA being $86 \mathrm{bp}$ from LSC/IRA border. For SSC/IRs, two copies of $n d h H$ straddle SSC/ IR borders, with $8 \mathrm{bp}$ of upstream copy and $1001 \mathrm{bp}$ of downstream copy moving into two sides of SSC region (except for $17 \mathrm{bp}$ of upstream and $1022 \mathrm{bp}$ of downstream copy in A. hybrida), and SSC $n d h F$ being $69 \mathrm{bp}$ from SSC/IRB border (except for $67 \mathrm{bp}$ in A. agardiana) (Additional file 15: Figure S4b).

\section{Repeat sequence analysis}

A total of 8234 (221 forward, 60 reverse, 192 palindromic and 7761 tandem) non-overlapped repeats were identified by using REPuter [30] and Phobos v.3.3.12 [31] for eleven Avena plastomes (Additional file 16: Figure S5a). Repeat numbers varied from 744 in A. sativa 

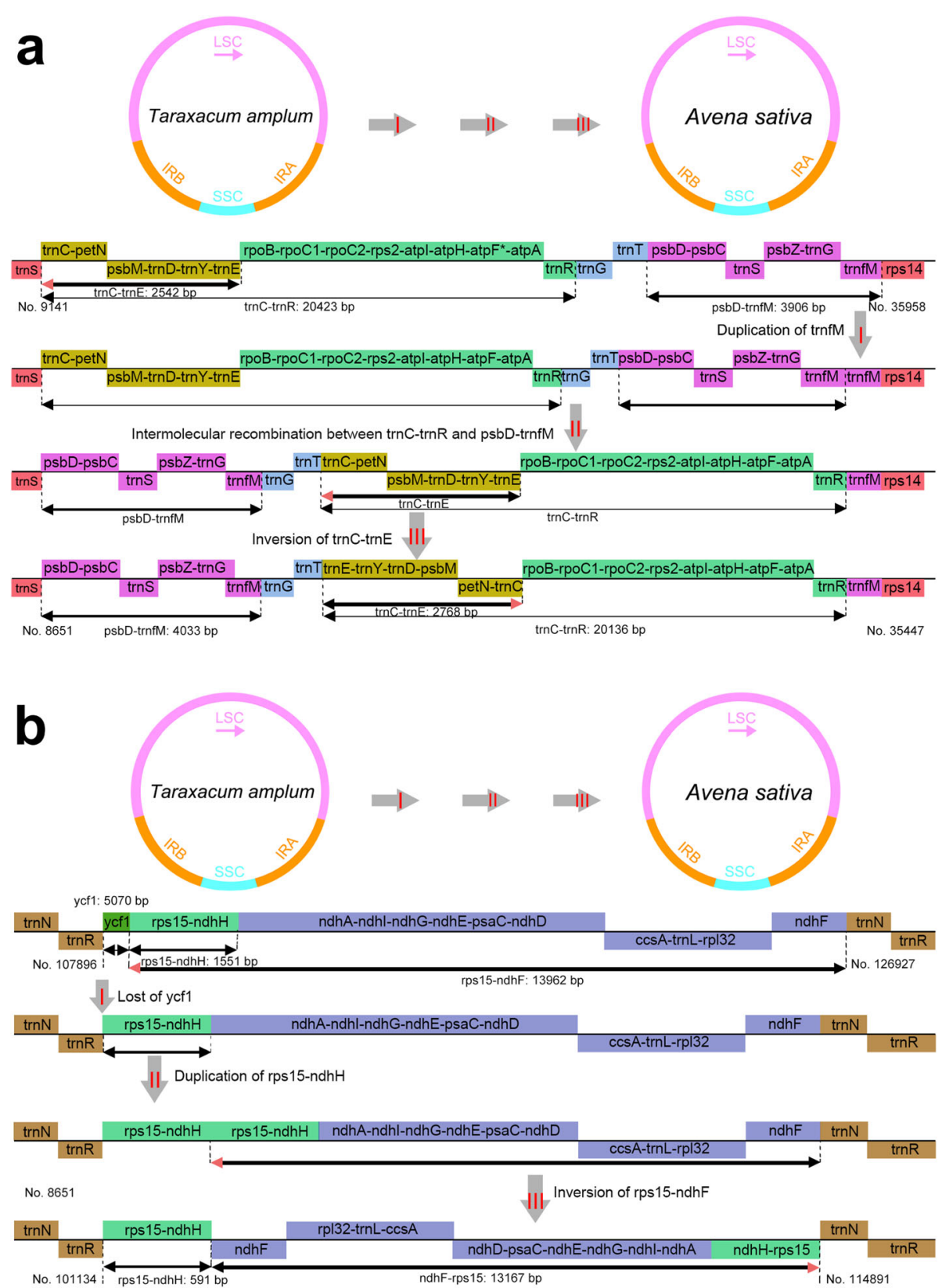

Fig. 3 Schematic diagram showing postulated intermolecular recombination events across Avena sativa (top) and Taraxacum amplum (bottom). a Gene order and orientation within LSC intermolecular recombination. Roman numeral arrows denote the sequences of recombination events: I, Duplication of trnfM gene; II, Intermolecular recombination of trnC-GCA-trnR-UCU and psbD-trnfM regions; II, Inversion of trnC-GCA-trnE-UUC region. b Gene order and orientation within IRB intermolecular recombination. Roman numeral arrows denote the sequences of recombination events: I, Loss of ycf1 gene; II, Duplication of rps 15-ndhH region; III, Inversion of rps 15-ndhF region. The vertical dashed lines indicate the rearrangement start-end base position. Gene transcribed in forward and reverse directions are indicated above and below the middle line, respectively

to 757 in diploids $A$. eriantha and $A$. ventricosa. Most abundant were tandem repeats (7-95 bp) ranging from 702 in A. sativa to 712 in A. eriantha and $A$. ventricosa, frequently with $7-10 \mathrm{bp}$ nucleotides (Additional file 4: Table S4a, S4b computed by REPuter [30] and Phobos [31]). Reverse repeats were the least abundant repeats, ranging from 3 in A. eriantha and
A. ventricosa to 11 in $A$. nuda, with $21-30$ bp nucleotides (Additional file 4: Table S4b). For repeat distribution, 484 of 745 repeats (64.97\%) were found in $A$. hirtula plastome LSC region, and 434 of 744 repeats (58.33\%) were found in A. sativa plastome intergenic spacers (Additional file 4: Table S4c, Additional file 16: Figure S5b). For the tandem repeat distribution, 455 


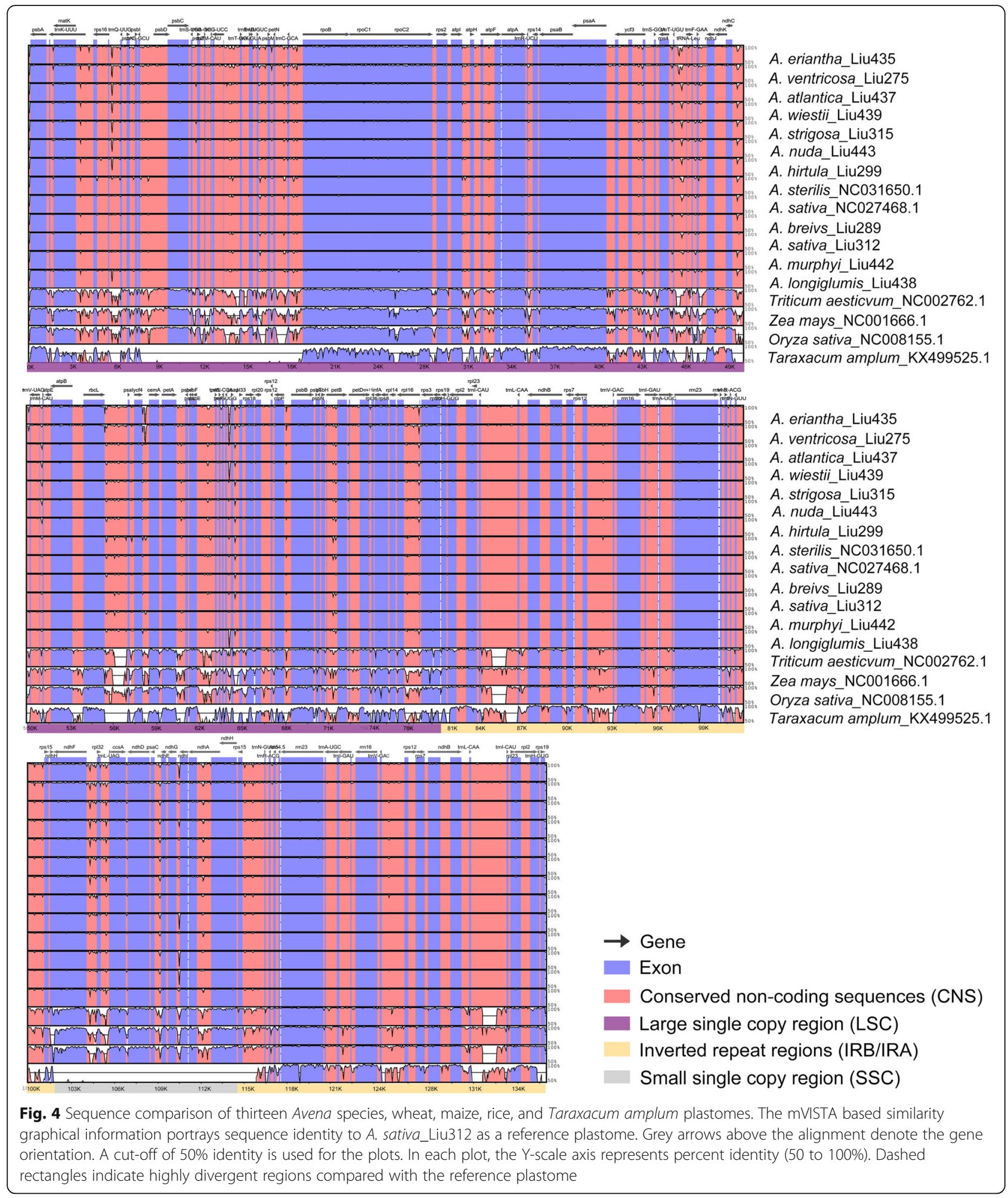

of 712 tandem repeats (64.63\%) were found in LSC region of A.ventricosa plastome, and 417 of 703 tandem repeats (59.32\%) were found in intergenic spacers of $A$. brevis plastome (Additional file 4: Table S4d, S4e, Additional file 16: Figure S5c).
There are 276 simple sequence repeats in Avena plastomes by Perlscript MicroSAtellite (MISA) [32], with the mononucleotide simple sequence repeats (SSRs) being the most abundant (Additional file 5: Table S5a). In each case, the 21-24 mononucleotide SSRs, one dinucleotide 

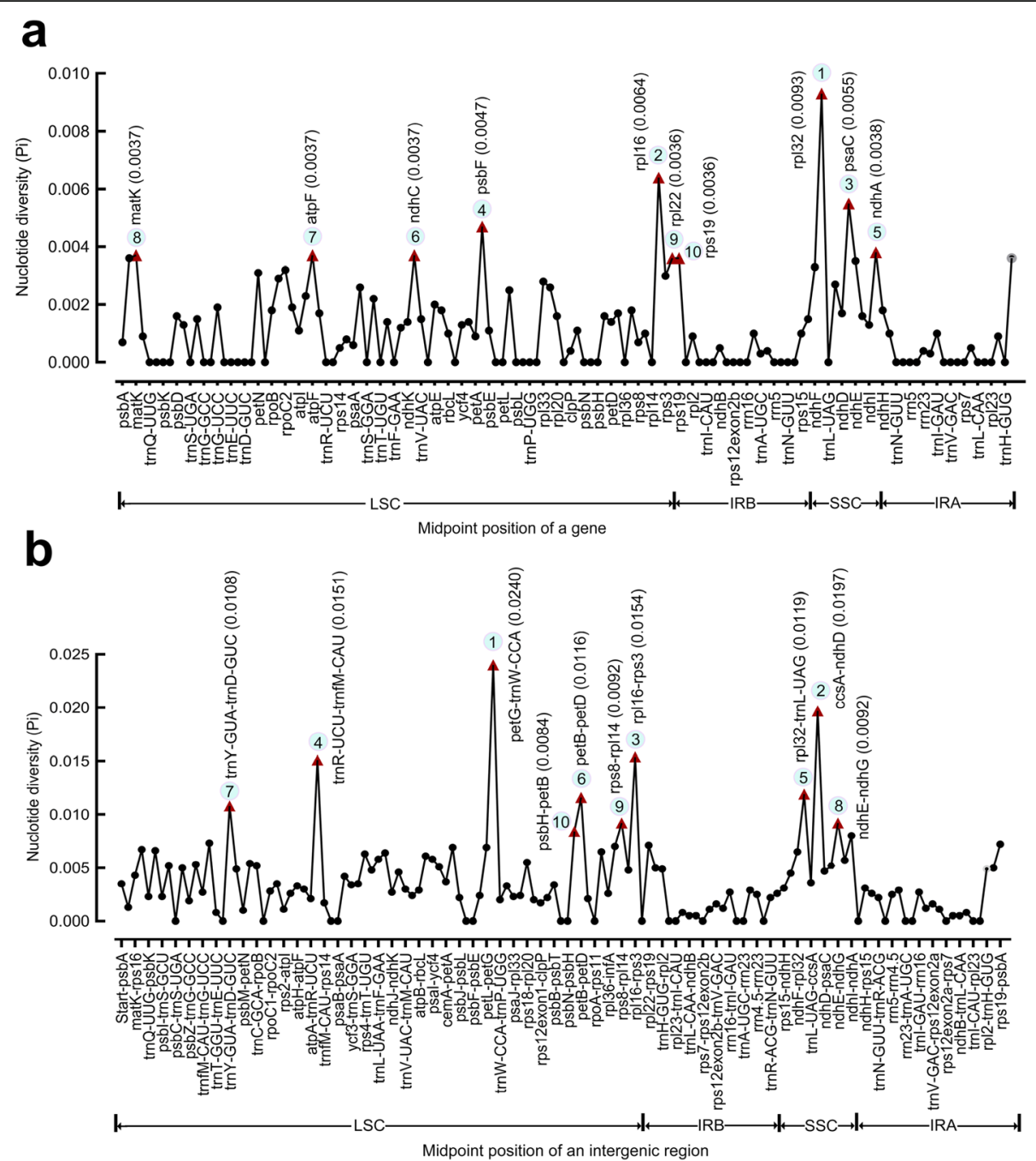

Fig. 5 Nucleotide diversity (Pi) values using the aligned Avena plastome of (a) ten most polymorphic single copy genes and (b) ten most polymorphic intergenic regions. Regions are oriented according to the midpoint positions in plastome sequences with top $10 \mathrm{Pi}$ values marked by red triangles

SSRs, and one-three composite SSRs are identified. No trinucleotide or tetranucleotide cpSSR exists in Avena. In general, the intergenic cpSSRs are more abundant than the genic cpSSRs in Avena, where the 15-20, fourfive, and two-six SSRs are found in the intergenic, coding, and intron regions respectively (Additional file 5: Table S5b, Additional file 17: Figure S6). This also occurs in Asteraceae [11], Orchidaceae [33] and Fabaceae [34], probably due to an associated lower polymorphism of coding regions in contrast to non-coding regions. The C-genome diploids $A$. eriantha or $A$. ventricosa plastomes harbour 24 mononucleotide SSRs (92.31\%) with 20 SSRs $(77.00 \%)$ in the intergenic regions (Additional file 17: Figure S6). SSRs are more abundant in LSC region than in SSC and IR regions (Additional file 5: Table S5a). The majority of mononucleotide SSRs $(17-20 ; 85-100 \%)$ are composed of $\mathrm{A} / \mathrm{T}$, which contributes to the base composition bias in Avena (A + T: 61.49-61.59\%; Additional file 2: Table S2).

We have visualized the extent to which 868 tandem repeats and 221 indel mutations are nonnormally distributed among Avena plastomes within $150 \mathrm{bp}$ windows (Additional file 7: Table S7) using $\mathrm{R}$ package circlize [35]. There are 81 insertions (66.94\%) and 57 deletions (57.00\%) located within tandem repeats (rings "c-e" in Fig. 6). We have explored the extent of genome-wide association between tandem repeats, indels and SNPs in Avena species alignments with $A$. sativa_Liu312 as a reference (Additional file 6: Table S6a, S6b). Due to the nonrandom distribution of mutation data in Avena plastomes, Spearman's Rho correlations are performed among tandem repeats, indels and SNPs (Table 4). All these correlations are observed with high significance $(p<0.01)$. The average of correlations is stronger 


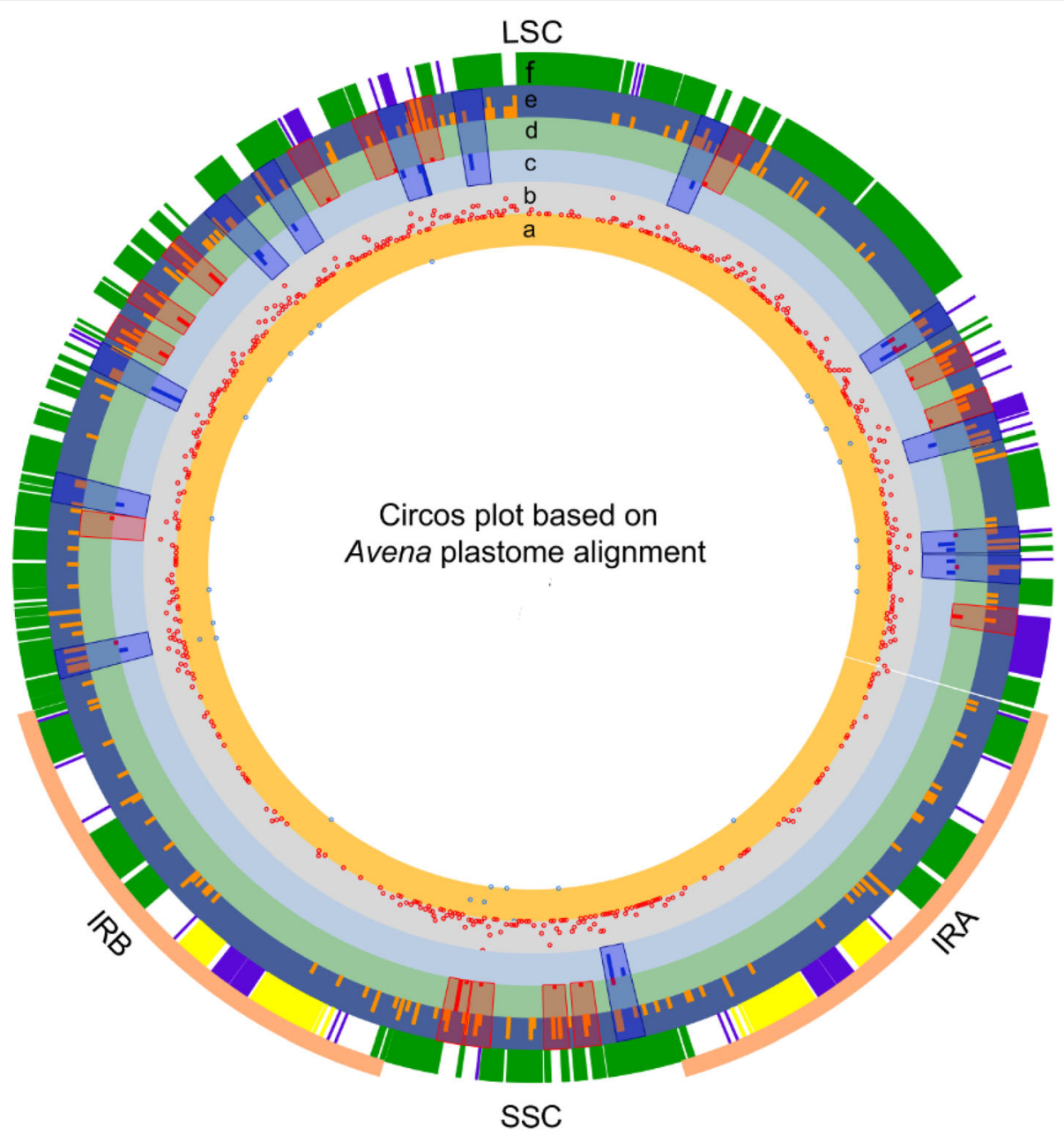

Fig. 6 Circos plot based on complete chloroplast genome alignment between eleven Avena species presented here available in Genome Warehouse database and two Avena species available in NCBI (NC031650.1 and NC027468.1) with A. sativa_Liu312 as a reference. All data in rings showing the location relationship between tandem repeats, insertions/deletions (indels) and single nucleotide polymorphisms (SNPs) by R package circlize [35] in the non-overlapping 150 bp bins. For plastome alignment, rings "a-b" show the location of substitutions and SNPS, respectively. Dots with a high relative positions in rings "a-b" represent more polymorphic loci in the 150 bp window. Rings "c-d" show the location of deletions and insertions, respectively. Ring "e" shows tandem repeat (7 bp-95 bp) location by Phobos [31] with parameters of repeat length $\leq 100 \mathrm{bp}$ and sequence identify $\geq 85 \%$. The relatively height of columns in rings " $\mathrm{c}-\mathrm{e}$ " represent the relative number of polymorphic loci belonging to deletions, insertions, or tandem repeats within the $150 \mathrm{bp}$ window, respectively. Ring "f" shows A. sativa_Liu312 chloroplast map with coding genes labeled in green, rRNAs in yellow and tRNAs in blue. Blue shadows denote the surrounding location of tandem repeats and deletions; Red shadows denote the surrounding location of tandem repeats and insertions. LSC, SSC, IRs denote large single-copy, small singlecopy, and inverted repeat regions of Avena plastome alignment. Total 702 tandem repeats, 141 insertions, 100 deletions, 992 SNPs, and 33 substitutions shown in the diagram

Table 4 Spearman's Rho correlation analysis result among tandem repeats, indels and SNPs using R v.3.5.3 [36] with correlation strengths of Akoglu [37] based on plastome alignments between eleven Avena species presented here available in Genome Warehouse database and two Avena species available in NCBI (NC031650.1 and NC027468.1) with A. sativa_Liu312 as a reference (150 bp windows)

\begin{tabular}{llll}
\hline & Tandem repeats and indels & Tandem repeats and SNPs & Indels and SNPs \\
\hline Rho & 0.3585 & 0.2607 & 0.2606 \\
$p$-value & $2.20 \times 10^{-16 * * *}$ & $1.48 \times 10^{-15 * * *}$ & $1.53 \times 10^{-15 * * *}$ \\
\hline
\end{tabular}

***Correlation was strongly significant at $p<0.01$ 
between tandems and indels, followed by tandems and SNPs then by indels and SNPs. The average value of correlations between tandems and indels is 0.3585 , between tandems and SNPs is 0.2607, and between indels and SNPs is 0.2606 in thirteen Avena species. MannWhitney $U$ test results show that a significant difference in the number of indels and SNPS from the tandem window and the non-tandem windows, with the corresponding $p$-vaules being $1.251 \times 10^{-9}$ and $1.477 \times 10^{-9}$, respectively.

\section{Gene selective pressure analysis}

All protein-coding genes went through purifying selection: only 27 coding genes have non-synonymous/synonymous mutation $(\mathrm{Ka} / \mathrm{Ks})$ ratio $<1$, with $\mathrm{Ka} / \mathrm{Ks}$ ratio ranging from 0.0313 (rps3) to 0.8896 (rpoC2) (Additional file 8: Table S8). The rps3 has no nonsynonymous rate change, and atpE, atpI, ndhD, ndhH-SSC, rbcL, rpl32, rpl33, rpoA, rps11, rps2, and ycf4 have no synonymous and non-synonymous changes (Additional file 18: Figure $\mathrm{S} 7)$. Ka/Ks ratios of photosynthesis loci including $p s b A(0.1354)$, petA (0.1416-0.2789), two subunits of ATP synthase genes (atpE and atpI) range from 0.1585 to $0.1632 \mathrm{Ka} / \mathrm{Ks}$ ratios of four subunits of $n d h$ genes ( $n d h D, n d h F, n d h H-S S C$ and $n d h I)$ range from 0.0353 to 0.2351 . $\mathrm{Ka} / \mathrm{Ks}$ ratios of self-replicating genes are as follows: $0.0313-0.2763$ of ribosomal protein small subunit genes (rps2, rps3, and rps11), 0.24050.2621 of ribosomal protein large subunit genes (rpl32 and rpl33); $0.0446-0.8896$ of DNA dependent RNA polymerase genes $(r p o A, r p o B, r p o C 1$ and rpoC2); and $0.1395-0.2840$ of infA. Ka/Ks ratios of other genes are
$0.2548-0.7669$ of matK, $0.1334-0.1775$ of $\operatorname{css} A$ and 0.1550 of $y c f 4$. The atpF, matK and rpoC2 genes in LSC region have a faster divergence than those genes in SSC or IRB regions. The rpoC2 gene is the fastest evolving gene in A-genome diploids $A$. brevis and A. longiglumis (Additional file 18: Figure S7). Coding genes in IRs (rps19, rpl2, rpl23, ndhB, rps7, rps12 and rps15) do not show Ka and/or Ks rate changes.

\section{Codon usage bias}

The protein-coding genes present a total of 19,913 codons in A. brevis plastome to 19,921 in A. eriantha and A. ventricosa plastomes (Additional file 8: Table S8a) with the RSCU (relative synonymous codon usage) values [38] ranging from 0.28 (CUG) to 2.12 (UUA) (Additional files 8, 9: Tables S8b, S9a, S9b, Fig. 7, Additional file 19: Figure S8). Leucine (10.76-10.78\% of each species) and cysteine (1.09\% of each species) are the most and the least abundant amino acids except for stop codons $(0.42 \%$ of each species; Additional file 9: Table S9b). Our findings match the trend reported across other angiosperm plastomes [7], which show leucine and isoleucine to be the most common codons. No codon bias can be shown by methionine (AUG) and tryptophan (UGG), encoded by only one codon. Codon usage is biased towards $\mathrm{A}$ and $\mathrm{T}$ at the third codon position (Additional file 10: Table S10a, S10b, Additional file 19: Figure S8). The AT content for first, second and third codons average 52.7, 60.3 and $70.0 \%$ in Avena (Additional file 8: Table S8a), respectively. Almost all A/Uending codons have RSCU values larger than one except for trnL-UAG and trnS-UGA (RSCU $=0.87,0.99$

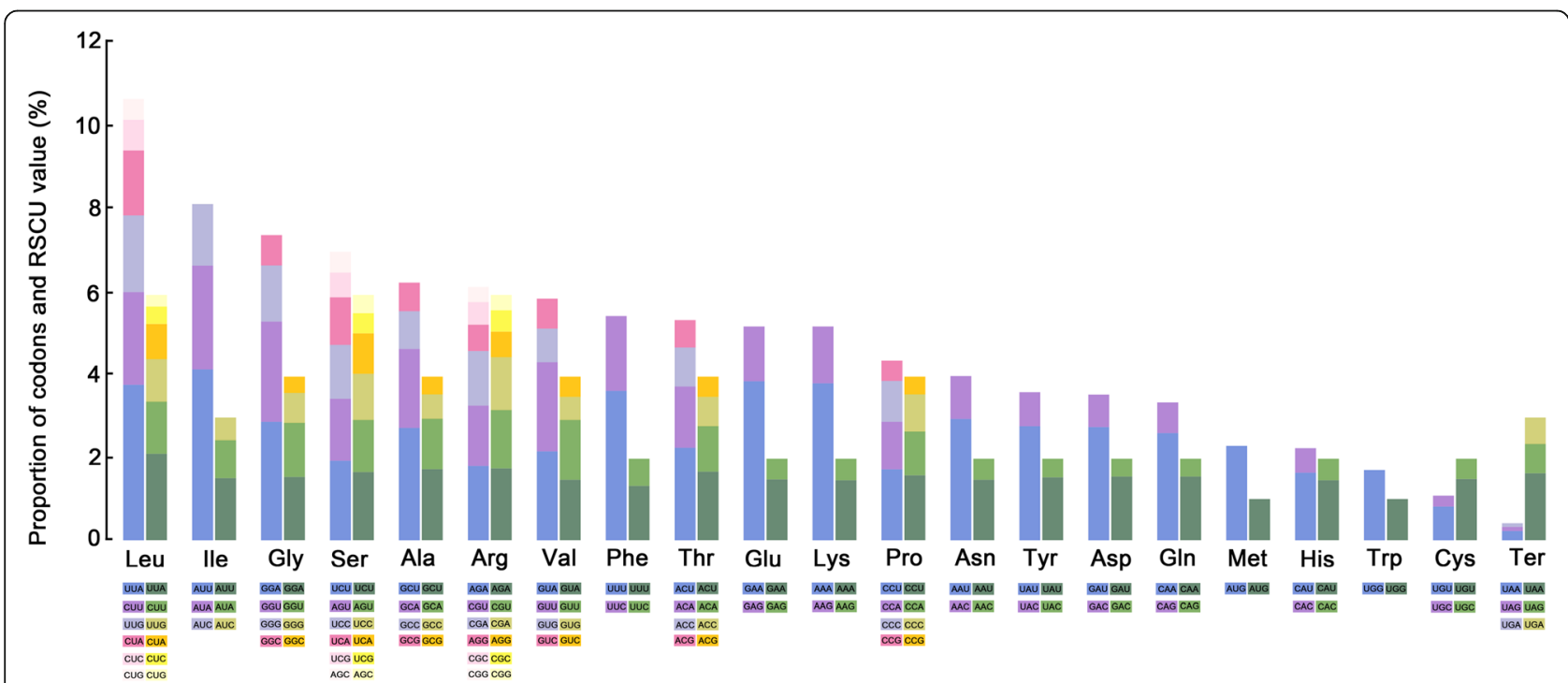

Fig. 7 Codon content of 20 amino acids and stop codons in protein-coding genes of Avena plastomes. The histogram on the left-hand side of each amino acid denotes codon usage within Avena plastomes, and the right-hand side denotes the codon RSCU [38] values. Colors correspond to codons listed underneath the columns 
respectively), whereas all of $\mathrm{C} / \mathrm{G}$-ending codons have RSCU values $\leq 1$ in Avena species (Additional file 11: Table S11a, S11b).

\section{Phylogenomic analyses}

Our phylogenomic analyses substantially increased resolution and provided robust phylogenetic relationships in Avena (Fig. 8). The monophyly of Avena received strong maximum likelihood bootstrap support (MLBS $=100 \%)$. Two strongly supported infrageneric lineages (MLBS = 100\%) are identified in Avena: clade I contains the Agenome diploid-polyploid subclade I (A. sativa, A. sterilis, $A$. murphyi, and $A$. brevis), subclade II (A. nuda, $A$. atlantica, $A$. hirtula, $A$. strigosa, and $A$. wiestii), and the basal position of $A$. longiglumis received strong support (MLBS $=100 \%$ ); and clade II contains the C-genome diploid lineage (A. eriantha and A. ventricosa) (Fig. 8).
Maximum likelihood (ML) topologies were constructed using not only the combined sequences of LSC and IRB intermolecular recombination fragments (28, $164 \mathrm{bp}, 14,262 \mathrm{bp}$ ), but also those of combined ten most polymorphic genes $(11,028 \mathrm{bp}, 8212 \mathrm{bp})$ and those of combined ten most polymorphic intergenic regions (3099 bp, 2374 bp) in order to develop suitable plastid markers for inferring the interspecific phylogenetic relationships (Additional file 20: Figure S9 [39-41]). Numbers above nodes are the trustable Shimodaira-Hasegawa test-approximate likelihood-ratio test support/Ultrafast bootstrap support (SH-aLRT > 80.0\%, UFBoot > 80.0\%). Phylogenetic tree of LSC intermolecular recombination fragments is consistent with complete plastome ML tree topology (Additional file 20: Figure S9a), showing the basal position of $A$. longiglumis within clade I with strong support $(\mathrm{SH}-\mathrm{aLRT}=100 \%$, UFBoot $=100 \%)$. The remaining topologies show that $A$. longliglumis is sister

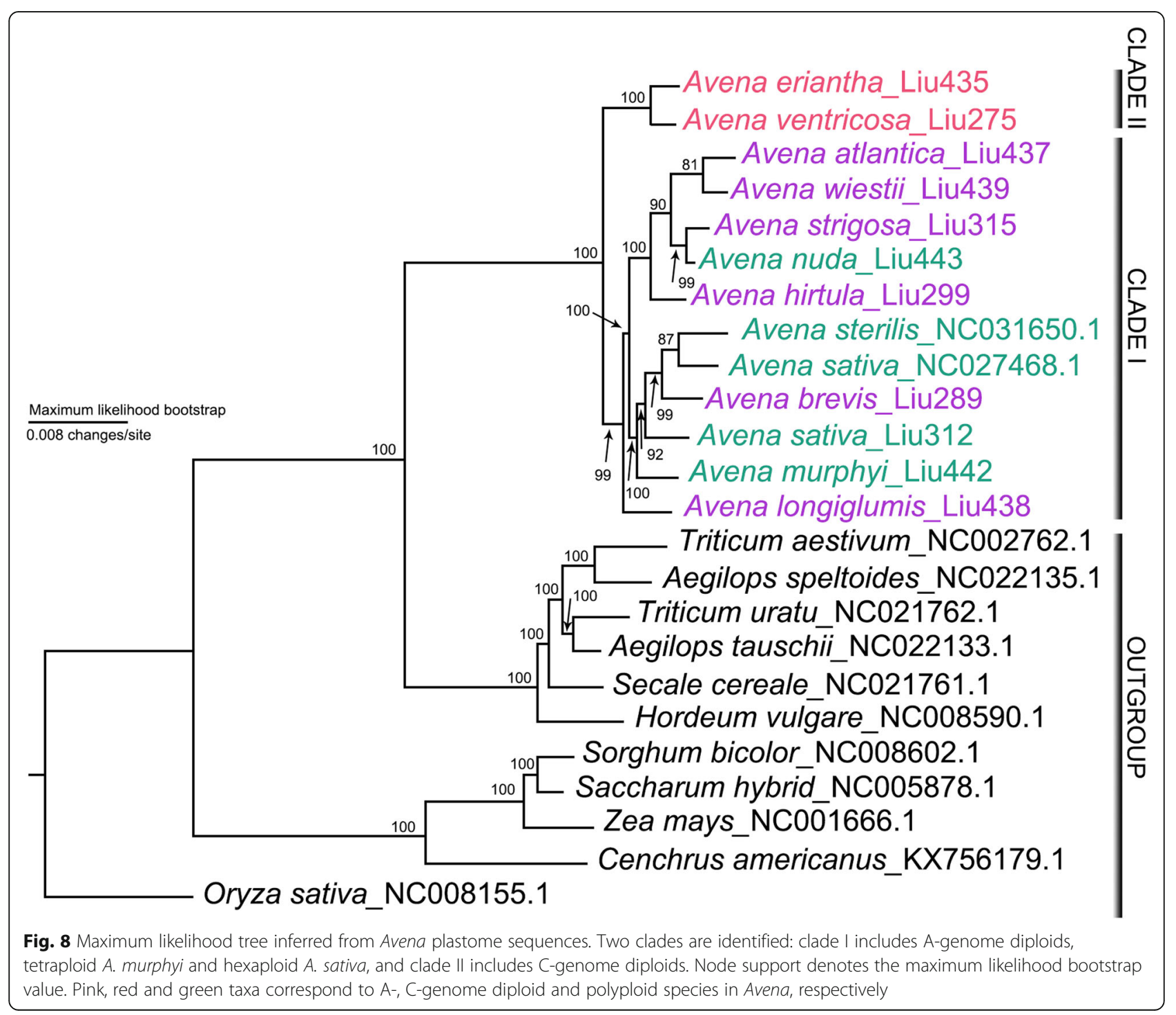


to subclade I with medium support $(\mathrm{SH}$-aLRT $=82.8 \%$, UFBoot $=86.0 \%$ ) by IRB intermolecular recombination fragments (Additional file 20: Figure S8b) with weak support (SH-aLRT $<50.0 \%$, UFBoot $<50.0 \%$ ) and by the phylogenetic topologies of protein-coding genes and intergenic regions with high nucleotide diversities (Additional file 20: Figure S9b-S9f). ML topology is further constructed using plastomes of Avena species presented here available in Genome Warehouse and 25 Avena species available in NCBI $[19,24]$. It is without significant topological change compared to a previous Bayesian maximum clade credibility (MCC) tree [19]. Namely, clade I contains the $A$. sativa inserted subclade I (from A. agadirianan to $A$. sterilis), the $A$. nuda inserted subclade II (from A. atlantica to $A$. nuda) and the basal position of $A$. longiglumis together with extra three diploid species (A. canariensis, $A$. damascene and $A$. lusitanica) in ML tree of 38 Avena plastomes (Additional file 21: Figure S10).

\section{Discussion}

\section{Plastome evolution}

All Avena plastomes possessed the typical gramineous plastome structure including the LSC and IRB intermolecular recombinations that are present in nearly all Poaceae, e.g. Oryza, Hordeum, Triticum, Secale, Cenchrus, Saccharum, Sorghum and Zea (Fig. 3). Liu et al. [11] suggested that the LSC recombination region has the gene (partially) missing in certain lineages, e.g. the missing $\operatorname{clp} P$ introns or rpoC1 intron, and this could be a particularly active region for sequence rearrangement in Poaceae plastomes [42]. Avena plastome average GC content $(38.48 \%)$ is similar to other monocot plastomes, such as Elodea canadensis (37\%) [43], Smilax china (37.25\%) [11], and Najas flexilis (38.2\%) [44]. According to report, 70 up to 88 protein-coding genes are present in angiosperm plastomes [45], there are 84 such genes in Avena plastomes. The $n d h$ gene size and content vary widely among some heterotrophic species, which retain photosynthetic ability due to the non-functional role of $n d h$ genes [44]. In the Asteraceous dandelion (Taraxacum amplum Markl. in T. officinale F.H. Wigg. agg.) plastome, rpoC1 is interrupted by a $\sim 680 \mathrm{bp}$ intron, and two exons expand for 450 and $1638 \mathrm{nt}$ respectively [12]. In oat plastome, the rpoC1 intron is absent. Intron excision from the primary transcript by splicing represents a prerequisite for translation of messenger RNAs (mRNAs) into the correct full-length protein, while the selective pressure of group II intron splicing events may have been important and their role underappreciated [46]. The rps12 is trans-spliced with exon 1 coded in LSC region and exons 2 and 3 in IRs. Spliceosomal introns are a feature of eukaryotic nuclear genes, and intron splicing can enhance gene expression [47]. Other aspects of mRNA metabolism, including pre-mRNA polyadenylation, editing transcription and mRNA decay are also influenced by removal of introns by the spliceosome [48].

The LSC/IRs and SSC/IRs borders are relatively conserved among angiosperm plastomes, mostly positioned within rps19 or $y c f 1$ [49]. Compared with the ancestral angiosperm genome structure (represented by Nicotiana tabacum [50]), the Avena plastome IRs have expanded into the LSC region, resulting in the movement of rps19. Significant expansions have been reported in other plants, such as the extreme $50 \mathrm{kbp}$ expansion found in Pelargonium $\times$ hortorum L.H. Bailey [51], and the $4 \mathrm{kbp}$ expansion in Jasminum nudiflorum Lindl [52].. Here, no significant IR length variation was detected among Avena plastomes.

It is well known that certain plastome regions show different mutation rates. Dispersed repeats may facilitate intermolecular recombination and plastome diversity creation, because the genome regions with increased sequence diversity could be formed by repeat sequence abundance in prokarya and eukarya [53]. The cpSSRs and indels are mainly distributed in the non-coding regions of Avena plastomes, the similar distribution preference of cpSSRs and indels has been reported in Olea europaea, Pseudoroegneria libanotica and Salvia miltiorrhiza [54-56].

The nonrandom distribution of tandem and mutation locations have been found in Avena plastome visualization (Fig. 6), and correlations at the interspecific level also exhibits variations. We observe moderate correlations between tandems and indels, and weak correlations between tandems and SNPs and between indels and SNPs in Avena plastomes. Comparisons of tandempresence-bin and tandem-absence-bin show strong difference for numbers of indels and SNPs, these differences are also statistically significant $(p<0.001)$ by Mann-Whitney $U$ test results. Araceae and Malvaceae plastomes have associations between repeats, indels and substitutions [21, 22], and the adjacent position relationships suggest a role for tandem repeats in SNP and indel mutations in Avena. These results support the hypothesis that tandem repeats play an important role in causing plastome mutations [21].

All protein-coding genes were found to be under purifying selection. This pattern has also been demonstrated in other Poaceae plastomes such as rice, maize and wheat [57], reflecting the typically conservative plastid genome across most angiosperms. Changes of nucleotide substitution rates have been correlated with obligate parasitism rather than loss of photosynthesis [58]. In parasites, there is less selective pressure on plastid function, so purifying selection on genes encoding proteins for DNA maintenance and expression may be relaxed. Increasing specialization on external carbon (e.g., 
improved nutrient acquisition efficiency) thus leads to changes of evolutionary rates of plastid housekeeping machinery because of the lifestyle, although with most plastid proteins being encoded in nucleus, the selection strength at protein-coding loci in plastids is unclear.

\section{Phylogenetic analysis in Avena}

To resolve relationships among closely related diploid species, it is imperative to identify rapidly evolving loci [11]. Concordant interspecific relationships are recovered by the phylogenies inferred from LSC intermolecular recombination fragment and from complete plastome sequences. There might be evolutionary convergence between the fragment from $p s b D$ to $\operatorname{trnR}-U C U$ and complete plastome sequences as a result of gene interaction and co-evolution to conserve the chloroplast functions. The complete plastome ML tree supports the basal position of $A$. longiglumis within clade I with strong support (MLBS $=100 \%$; Fig. 8), and those highly supported within the A-genome diploid-polyploid lineage in previous studies $[19,24]$ and within clade I of ML tree of 38 Avena plastomes (Additional file 21: Figure S10). Diploid A. longiglumis together with A. canariensis, A. damascena and $A$. lusitanica are proposed to be oat potential ancestors $[19,24]$, whose contributions for oat speciation are deserve further investigation in the context of genomics and cytogenetic data.

Plastome marker selection should be made based on appropriate evolutionary rates ( $\mathrm{Pi}$ values) are appropriate. For subclade I, A. sativa is sister to diploid A. brevis and tetraploid $A$. murphyi based on the ten most polymorphic coding regions and non-coding regions respectively (Additional file 20: Figure S8c, S8d). Such variation indicates that $A$. brevis and $A$. murphyi might carry a diverged A-genome from the most likely A-genome diploid ancestor, A. longiglumis, supported by nuclear gene Pgk1 too [23]. It is also evident that the most frequently used chloroplast markers (including trnL-trnF and matK) show few polymorphisms $(0.0058,0.0037)$ at the interspecific level with respect to adding outgroup wheat $(0.0166,0.0136)$. The nucleotide diversity for 30 most polymorphic intergenic regions ranged from 0.0287 to 0.1100 in Hibiscus (Malvaceae) [59], substantially higher than in Avena top 30 intergenic regions with nucleotide diversity ranging from 0.0053 to 0.0240 . Eleven loci, including petG-trn $W-C C A, \operatorname{ccs} A-n d h D$, rpl32-trnL-UAG, trnY-GUA-trnD-GUC, rps16-trnQ-UUG, infA-rps8, petA-psbJ, trnF-GAA-ndhJ, rps4-trnT-UGU, ndhG-ndhI and $n d h F-r p l 32$, are shared by the two genera. Among the 10 loci, rpl32-trnL-UAG is also shared by Fritillaria [60], and rps16-trnQ-UUG is also shared by Artemisia and Dendrobium $[61,62]$. In addition, $c c s A-n d h D, n d h F-$ rpl32, psbK-psbI are shared by Avena and Artemisia [61], and petN-trnC-GCA, rps12-clpP, and petL-petG are shared by Avena and Monsteroid species [63]. These markers could be used for the deep divergence in the family level as mutational hotspots but not for Avena.

\section{Conclusion}

Diversification of Avena plastomes is explained by the presence of highly diverse genes and intergenic regions, LSC intermolecular recombination, and the cooccurrence of tandem repeats and indels or single nucleotide polymorphisms. The study demonstrates that the A-genome diploid-polyploid lineage maintains two subclades derived from maternal ancestors, and the diverged A-genomes originate from the diploid A. longiglumis, the optimum candidate ancestor for the Agenome in polyploid species. The genus does deserve attention as a model system to understand the underlying mechanisms of plastome evolution, because of the need to mine genetic resources from both chloroplast and nuclear genomes for crop variety breeding.

\section{Methods \\ Isolation of DNA and sequencing}

Seeds of eleven Avena species (sample origin and genome designation [16] given in Additional file 1: Table S1) were obtained from CN-Saskatchewan and USDABeltsville Germplasm System in this study. Healthy leaf samples were collected from South China Botanical Garden Greenhouse in Guangzhou, China. Whole genomic DNA was extracted from $100 \mathrm{mg}$ fresh leaf tissue using the DNeasy Plant Mini Kit following the manufacturer's protocol (Biomed, Beijing, China).

DNA libraries were prepared and sequenced with the Illumina HiSeq2500 platform (Illumina, San Diego, CA, USA) for A. brevis, A. hirtula, A. strigosa and A. sativa with PE250 bp reads from 300 bp insert libraries and the remaining seven species with PE250 bp reads from 500 bp insert libraries. Project data have been deposited at the Genome Sequence Archive (GSA) of National Genomics Data Center with accession number CRA003107 (https://bigd.big.ac.cn/search/?dbId=\&q=CRA003107).

Three coding gene sequences with the highest coverage were used the seed sequence for de novo assembly of Avena plastome by NOVOPlasty v.2.6.2 [64] with $A$. sativa (NC027468.1) as a reference for IR regions correction (Fig. 1). Then Geneious R11 [65] was used for contigs merging and de-redundancy. Chloroplast circularization and initiation site determination were manually processed.

\section{Plastome assembly and annotation}

Many protocols are available for chloroplast genome assembly including SOAPdenovo2 [66] and Velvet [67]. Velvet is suitable for small genome assembly, and we found SOAPdenovo2 and Velvet cannot independently 
assemble one complete plastome sequences of Avena without auxiliary gap-filling softwares. Since NOVOPlasty v.2.6.2 [64] is capable of extending one read into a complete circular genome through extending the given seed to form circular genome, three single copy coding gene sequences with the highest coverage were used as starting seed sequences for de novo assemblies of Avena plastomes by NOVOPlasty separately with k-mer values of 79, 89, 99 and 109 (Fig. 1). Chloroplast cyclization and initiation site determination were optimized manually. The integrity of Avena plastomes was also verified by Velvet contigs covered $99 \%$ of the NOVOPlasty assemblied plastomes. Average coverage depth $(1634 \times$ to $5339 \times)$ was calculated by Geneious R11 [65] by mapping the total clean reads to de novo assembled plastome of each species (Table 1, Additional file 12: Figure S1).

The chloroplast genes were annotated by GeSeq [68] with default parameters to predict protein-coding genes, rRNA and tRNA genes (Tables 2, 3, Additional file 13: Figures S2). IRs were confirmed by IR finder [69]. All tRNA genes were further verified by using tRNAscan-SE (http://lowelab.ucsc.edu/tRNAscan-SE/). GC content was calculated by Geneious R11 [65]. The circular chloroplast genome map was drawn by OrganellarGenomeDRAW (OGDRAW) v.1.3.1 [26] followed manual optimization (Fig. 1). Plastomes were submitted to Genome Warehouse in National Genomics Data Center (Additional file 1: Table S1).

\section{Complete chloroplast genome comparison}

The data gave maximum coverage depth of $2643 \times$ to $9373 \times$ for Avena plastomes (Additional file 12: Figure S1). Chloroplast genome similarity of eleven Avena species, wheat, maize, and rice were assessed using BLASTN by GView with $10 \mathrm{kbp}$ connection windows [25]. The plastomes of eleven Avena species, two accessions of A. sativa, gramineous outgroups and Taraxacum amplum were respectively aligned by Mauve [27] in order to investigate intermolecular recombination events (Figs. 2, 3).

Plastome structures among Avena species, wheat, maize, rice, and dandelion were compared by mVISTA percent identity plot in Shuffle-LAGAN mode [28, 70] to reveal several major genomic variations located in LSC and SSC regions (Fig. 4). Subsequently, nucleotide diversity of single copy genes and intergenic regions was estimated for two sequence datasets (thirteen Avena species+Triticum aestivum plastomes) by DnaSP v.6 [29] (Additional file 4: Table S4, Fig. 5). Plastome genetic architecture of eleven Avena species available in Genome Warehouse, Triticum aestivum plastomes and fourteen Avena species available in NCBI for LSC/IRs and SSC/IRs borders were analysed by IRscope [71] (Additional file 15: Figure S4).

\section{Repeat structure identification}

Plastome repeat sequences were identified by REPuter [30] (repeat unit length minimum $\geq 21 \mathrm{bp}$, repeat identity $\geq 90 \%$, Hamming distance 2). Four matches of repeats were classified as follows: (i) forward, (ii) reverse, (iii) complement, and (iv) palindromic match (Additional file 4: Table S4a, S4b, S4c, Additional file 16: Figure S5a, S5b, S5c). Tandem repeats (13-75 bp) were identified by using Phobos v.3.3.12 [31] (Additional file 4: Table S4d, S4e). Simple sequence repeats (SSRs) were examined by Perlscript MicroSAtellite (MISA) [32] with parameters setting as follows. The motif sizes were one to six nucleotides, and the minimum repeat unit was defined as 10 for mononucleotides, six for dinucleotides, and five for tri-, tetra-, penta-, and hexa-nucleotides (Additional file 5: Table S5a, S5b, Additional file 17: Figures S6). Repeat type and distribution were analysed by GraphPad Prism v.8.0. Selecting A. sativa_Liu312 plastome as a reference for coordinate positions, tandem repeats were detected by Phobos [31], and indels and SNPs were counted within the non-overlapping $150 \mathrm{bp}$ window for thirteen Avena plastomes using Mauve pairwise alignment (Additional file 6: Table S6a, S6b).

Circos plot, showing the locations and relationships between tandem repeats, insertions/deletions (indels), and single nucleotide polymorphisms (SNPs), was generated by $\mathrm{R}$ package circlize [35]. Comparative plastome studies show that certain plastome regions are predisposed to mutations due to nonrandom distribution of tandem and mutation locations (Fig. 6), so Spearman's Rho correlation analyses are performed to measure the degree of association between tandems and mutations and between mutations by $\mathrm{R}$ v.3.5.3 [36]. The strengths of Spearman rank correlations between two variables have set threshold values as follows: negligible or very weak (0.1-0.19), weak (0.20-0.29), moderate $(0.30-0.39)$ and strong (0.4-0.69) [37]. The probability $(p)$ of significance of the correlations was tested at $\alpha$-level of 0.01. MannWhitney $\mathrm{U}$ tests are further performed to decide whether tandem repeat presence/absence affects the numbers of indels and SNPs in each 150 window by R v.3.5.3 (Additional file 7: Table S7), using grouping variables (tandem repeat number $>0$ or $=0$ ) and outcome variables (indel number and SNP number) in each $150 \mathrm{bp}$ window.

\section{Synonymous codon usage bias}

To investigate the selective pressure on plastome proteincoding genes between two species, $\mathrm{Ka} / \mathrm{Ks}$ values were calculated by KaKs_Calculator 2.0 [72] (Additional file 8: Table S8a, Additional file 18: Figure S7). RSCU values were examined by CodonW v.1.4.2 [38]. Codon absolute number and codon usage patterns were analysed by GraphPad Prism v.8.0 (Additional files 9, 10, 11: Tables S9, S10, S11; Fig. 7, Additional file 19: Figure S8). 


\section{Phylogenomic analysis}

Phylogenetic trees were constructed by ML analyses using Avena and gramineous outgroup plastome sequences $(162,505 \mathrm{bp})$ based on recent phylogeny [73]. The sequences were aligned and then manually adjusted by BioEdit [74]. The best-fitting nucleotide substitution model was determined using the Akaike Information Criterion (AIC) in iModeltest v.2.1.10 [75]. The GTR + $\mathrm{I}+\mathrm{G}$ model was used in ML analyses, which were performed by MEGA v.6.0 with 1000 bootstrap replicates [76].

In order to evaluate the alternative hypotheses of phylogeny, ML trees were constructed using not only the combined sequences of LSC or SSC intermolecular rearrangement fragments $(28,164 \mathrm{bp}, 14,262 \mathrm{bp})$, but also those of combined ten most polymorphic protein-coding genes $(11,028 \mathrm{bp}, 8212 \mathrm{bp})$ and combined ten most polymorphic intergenic regions (3099 bp, 2374 bp) (Table S4, Fig. 8, S8) by IQ-TREE v.1.6.8 [39] employing the model-testing function to infer the best-fit substitution model for such six datasets under the Bayesian information criterion. To evaluate node reliability, we implemented a Shimodaira-Hasegawa-like approximate likelihood ratio test (SH-aLRT) [40], and branch support was assessed using Ultrafast Bootstrap Approximation (UFB) [41] using 1000 bootstrap replicates for each method. FigTree v.1.4.4 (http://tree.bio.ed.ac.uk/software/figtree/) was used to visualize ML trees (Additional file 20: Figure S8). For phylogenetic analyses of oat and its diploid wild species, the plastome sequences of 25 published Avena species were downloaded from NCBI [24]. ML tree was constructed using above procedure (Additional file 20: Figure S10).

\footnotetext{
Abbreviations

AIC: Akaike information criterion; BI: Bayesian inference; BLAST: Basic local alignment search tool; CDS: Protein-coding sequences; $\mathrm{cp}$ : Chloroplast: cpSSRs: Chloroplast simple sequence repeats; CTAB: Cetyl trimethylammonium bromide; C-terminal: Carboxy-terminal; DnaSP: DNA sequences polymorphism; GC: Guanine-cytosine; GTR: General time reversible; IGS: Intergenic sequences; Indel: Insertions/deletions; IR: Inverted repeat; IRA: Inverted repeat A; IRB: Inverted repeat B; IRs: Inverted repeat regions; Ka/Ks: Non-synonymous/synonymous mutation ratio; LSC: Large single copy; ML: Maximum likelihood; MLBS: Maximum likelihood bootstrap support; mRNAs: Messenger RNAs; NCBI: National Center for Biotechnology Information; N-terminal: Amino terminal; OGDRAW: OrganellarGenomeDRAW; Pi: Nucleotide diversity; rRNAs: Ribosomal RNAs; RSCU: Relative synonymous codon usage; SH-aLRT: Shimodaira-Hasegawa test-approximate likelihoodratio test support; SNP: Single nucleotide polymorphism; SSC: Small single copy; SSRs: Simple sequence repeats; tRNAs: Transfer RNAs; UFBoot: Ultrafast bootstrap support
}

\section{Acknowledgements}

We thank National Supercomputing Center in Guangzhou for data analyses.

\section{Authors' contributions}

QL, TS and JSHH conceived and designed experiments; MZL and WKX assembled and annotated the genome; QL, MZL and WKX analysed data; XYL, MZL and WKX performed experiments; QL, XYL, TS and JSHH wrote the paper. The authors read and approved the final manuscript.

\section{Funding}

This work was funded by Special Basic Research Foundation of Ministry of Science and Technology of People's Republic of China (2013FY112100), the China Scholarship Council Awards to Q. Liu (202004910143), and Overseas Distinguished Scholar Project of SCBG to J.S. Heslop-Harrison (Y861041001). The funders had no role in study design, data collection and analysis, decision to publish, or preparation of the manuscript.

\section{Availability of data and materials}

The complete chloroplast genomes generated in current study are released in the Genome Warehouse Database of National Genomics Data Center (https://bigd.big.ac.cn/search/?dbld=gwh\&q=PRJCA003205) with accession numbers GWHAOPA01000000- GWHAOPK01000000 and in the NCBI database (https://www.ncbi.nlm.nih.gov/) with GenBank accession numbers MK336388-MK336398. The illumina datasets analysed during the current study are available in the Genome Sequence Archive (GSA) of National Genomics Data Center with accession number CRA003107 (https://bigd.big. ac.cn/search/?dbld=\&q=CRA003107). All data generated or analysed during this study are included in this published article and the supplementary information files.

\section{Ethics approval and consent to participate}

We complied with all relevant institutional, national and international guidelines with permissions from South China Botanical Garden, Chinese Academy of Sciences.

\section{Consent for publication}

Not applicable.

\section{Competing interests}

The authors have declared that no competing interests exist.

\section{Author details}

${ }^{1}$ Key Laboratory of Plant Resources Conservation and Sustainable Utilization / Guangdong Provincial Key Laboratory of Applied Botany, South China Botanical Garden, Chinese Academy of Sciences, Guangzhou, China. ${ }^{2}$ Center for Conservation Biology, Core Botanical Gardens, Chinese Academy of Sciences, Guangzhou, China. ${ }^{3}$ University of Chinese Academy of Sciences, Beijing, China. ${ }^{4}$ Independent Researcher, Guangzhou, China. ${ }^{5}$ Department of Genetics and Genome Biology, University of Leicester, Leicester LE1 7RH, UK.

Received: 21 November 2019 Accepted: 25 August 2020

Published online: 02 September 2020

\section{Supplementary information}

Supplementary information accompanies this paper at https://doi.org/10. 1186/s12870-020-02621-y.

Additional file 1: Table S1. List of Avena species and their accession numbers in NCBI (or Genome Warehouse) included in the phylogenetic analyses of complete chloroplast genomes.

Additional file 2: Table S2. Characteristics of chloroplast genomes of analysed Avena species.

Additional file 3: Table S3. Nucleotide diversity (Pi) analyses of 13 Avena species (two congeneric species download from NCBI) and Triticum aestivum_NC002762.1 plastomes computed by DnaSP v.6 [29]. a Pi values of 131 plastid genes. The rps 12 is treated as three parts as the second gene divided into two independent transcription units. b $\mathrm{Pi}$ values of 130 intergenic regions.

Additional file 4: Table S4. Repetitive motif abundance in eleven Avena species and dandelion (Taraxacum amplum) plastomes computed by REPuter [30] and Phobos [31]. a Repetitive motif abundance computed by REPuter. F, P, R and C indicate the repeat types forward, palindrome, reverse and complement repeat, respectively. b Repeat type statistic analysis computed by REPuter. F, P, R and C indicate the repeat types forward, palindrome, reverse and complement, respectively. c Repeat distribution statistic analysis. LSC, SSC, IR, IGS and CDS indicate large single-copy, small single-copy, inverted repeat regions, intergenic 
and protein-coding sequences, respectively. $\mathbf{d}$ Tandem repeat abundance computed by Phobos. e Tandem repeat distribution statistic analysis.

Additional file 5: Table S5. Simple sequence repeat (SSR) sequences in eleven Avena species plastomes computed by Perlscript MicroSAtellite (MISA) [32]. a SSR sequence abundance. b SSR sequence distribution. IGS: intergenic sequences; CDS: protein-coding sequences; p1: mononucleotide repeat; p2: dinucleotide repeat; p3: trinucleotide repeat; p4: tetranucleotide repeat; c: composite SSR

Additional file 6: Table S6. Statistics between tandem repeats, indels and SNPs of plastome alignments between eleven Avena species presented here available in Genome Warehouse database and two Avena species available in NCBI (NC031650.1 and NC027468.1) with A. sativa_Liu312 as a reference. (a) Tandem repeats detected in Avena sativa_312 chloroplast genome using Phobos [31]. (b) Information of indels and SNPs in the complete chloroplast genome alignments.

Additional file 7: Table S7. The number and presence statistics of tandem repeats, indels and SNPs of plastome alignments between eleven Avena species presented here and two Avena species available in NCBI (NC031650.1 and NC027468.1) with A. sativa_Liu312 as a reference within each $150 \mathrm{bp}$ window.

Additional file 8: Table S8. Maximum likelihood parameter estimates and substitutions for the 27 plastid genes (atpA, atpE, atpF, atpl, ccs A, infA, matK, ndhA, ndhB, ndhD, ndhF, ndhH, ndhl, petA, psbA, rbcL, rpl2, rpl32, rp/33, rpoA, rpoB, rpoC1, rpoC2, rps2, rps3, rps11, and y(f4).

Additional file 9: Table S9. GC percent and codon usage for Avena plastomes computed by CodonW v.1.4.2 [38]. a Genomic GC percent and codon usage statistic analysis. $\mathbf{b}$ The third codon usage statistic analysis.

Additional file 10: Table S10. Codon usage preference of all protein coding genes for eleven Avena plastomes calculated by http://www. bioinformatics.org/sms2/codon_usage. a Codon numbers of 20 amino acids and stop codons. b Codon number percent (\%) of 20 amino acids and stop codons

Additional file 11: Table S11. The relative synonymous codon usage (RSCU) values for eleven Avena species computed by CodonW v.1.4.2 [38]. a RSCU values. b RSCU values subtracted by 1.00 (the expected value if no codon bias).

Additional file 12: Figure S1. Clean reads mapping to the assembled plastome of eleven Avena species. a $A$. atlantica $\mathbf{b} A$. brevis. c $A$ eriantha. d $A$. hirtula. e A. longiglumis. f $A$. murphyi. g A. nuda. h A. sativa. i $A$. strigosa. $\mathbf{j}$ A. ventricosa. $\mathbf{k}$ A. wiestii. The enrichment of $A$. brevis, $A$. hirtula, A. strigosa and A. sativa with PE250 bp reads from 300 bp insert libraries displays uniformity. It is better than those of the remaining seven species with PE250 bp reads from 500 bp insert libraries, whose reads enrichment displays valleys or peaks. There is no gap region along plastome sequences with the maximum coverage depth being $3004 x$ to $9373 x$.

Additional file 13: Figure S2. The intron sequence deletion of $r p o \mathrm{Cl}$ gene of Avena atlantica (2049 bp; GWHAOPC01000000) and Triticum urartu (2052 bp; NC021762.1) compared to Taraxacum amplum (2746 bp: KX499525.1). a The 688 bp intron deletion marked by two black arrows in alignment sequences. b-n The rpoC1 sequence alignment 1-2746 bp of A. atlantica, Triticum urartu and Taraxacum amplum.

Additional file 14: Figure S3. The rpoCl amino acid sequence alignments for Avena species, gramineous outgroups and Taraxacum amplum [11]. Amino acids that were conserved within Avena or among outgroup are colored according to physicochemical properties based on hydrophobicity color scheme. Black arrows indicated Avena-specific mutation.

Additional file 15: Figure S4. Comparison of border distance between adjacent genes and junctions of the LSC, SSC and two IR regions among (a) eleven Avena species and Triticum aestivum plastomes, and (b) fourteen Avena species plastomes available in NCBI. The adjacent border genes are denoted by colored boxes. The gaps between genes and the borders are denoted by the base pair (bp) lengths. The figure is not to scale with respect to sequence length and only shows relative changes near the IR/SC borders
Additional file $\mathbf{1 6}$ Figure S5. Repetitive motif abundance in eleven Avena and Taraxacum amplum plastomes (a) computed by REPuter [30]. $F, P, R$ and $C$ indicate the repeat types forward, palindrome, reverse and complement, respectively, and (b) computed by REPuter [30], to identify repeat sequences with length $\geq 21 \mathrm{bp}$ and sequence identify $\geq 90 \%$. c Tandem repeat distribution patterns by Phobos [31]. LSC, SSC, IR, IGS and CDS indicate large single-copy, small single-copy, inverted repeat regions, intergenic and protein-coding sequences, respectively.

Additional file $\mathbf{1 7}$ Figure S6. Visualization of simple sequence repeat (SSR) sequence distribution pattern in eleven Avena plastomes. IGS, CDS, $c, p 1$ and p2 indicate intergenic regions, protein-coding sequences, composite SSR, mononucleotide repeat and dinucleotide repeat sequences, respectively

Additional file 18 Figure S7. Gene-specific $\mathrm{Ka}$ and $\mathrm{Ka} / \mathrm{Ks}$ values between Avena plastomes. Ka, nonsynonymous rate; Ks, synonymous rate. Black solid dots denote gene-specific Ka/Ks values greater than 0.4000 .

Additional file 19 Figure S8. Visualization of the relative synonymous codon usage (RSCU) [38] patterns in eleven Avena species. The RSCU values are subtracted by 1.00 (the expected value if no codon bias). The color scale indicates the magnitude of overall RSCU values: The greenest codons are the most preferred, and the reddest the least preferred. C/Gending codons are in red, and A/T-ending codons are in blue.

Additional file $\mathbf{2 0}$ Figure S9. Maximum likelihood trees of 13 Avena species and Triticum aestivum based on the plastome matrix from two recombination hotspots, the combined ten most polymorphic genes, and ten most polymorphic intergenic regions. a ML tree based on combined LSC intermolecular recombination fragment sequences from $p s b D$ to $\operatorname{trnR}$. b ML tree based on combined IRB intermolecular recombination fragment sequences from $n d h F$ to $r p s$ 15. c ML tree based on combined ten most polymorphic gene sequences (rp/32, rp/16, psaC, psbF, ndhA, ndhC, atpF, matK, rpl22, and rps19) of 13 Avena species. d ML tree based on combined ten most polymorphic intergenic sequences (petG-trnW-CCA, cCSA-ndhD, rp/16-rps3, trnR-UCU-trnfM-CAU, rpl32-trnL-UAG, petB-petD, trnY-GUA-trnD-GUC, ndhE-ndhG, rps8-rp/14 and psbH-petB) of 13 Avena species. e ML tree based on combined ten most polymorphic gene sequences (matK, rp/32, trnfM-CAU, trnK-UUU, rpl16, ndhA, psaC, psbF, ndhF and rpl22) of 13 Avena species and Triticum aestivum. $\mathbf{f} \mathrm{ML}$ tree based on combined ten most polymorphic intergenic sequences (petG$\operatorname{trnW}$-CCA, cCSA-ndhD, rpl16-rps3, trnR-UCU-trnfM-CAU, rp/32-trnL-UAG, petBpetD, trnY-GUA-trnD-GUC, ndhE-ndhG, rps8-rp/14 and psbH-petB) of 13 Avena species and Triticum aestivum. Node numbers denote as: IQ-TREE [37] Shimodaira-Hasegawa test-approximate likelihood-ratio test support/ Ultrafast bootstrap support (SH-aLRT support/UFBoot support) [38, 39].

Additional file 21 Figure S10. Maximum likelihood tree inferred from complete chloroplast genomes of eleven Avena species presented here available in Genome Warehouse database and 25 Avena species available in NCBI. Triticum aestivum is used as outgroup. Two clades are identified: clade I includes the $A$. sativa inserted subclade I (from $A$. agadirianan to A. sterilis), the A. nuda inserted subclade II (from A. atlantica to A. nuda) and the basal position of $A$. longiglumis together with extra three diploid species (A. canariensis, A. damascene and A. lusitanica), and clade II includes $\mathrm{C}$-genome diploids. Node support denotes the maximum likelihood bootstrap value. Pink, red and green taxa correspond to A-, Cgenome diploid and polyploid species in Avena, respectively.

\section{Author details}

Supplementary information accompanies this paper at https://doi.org/10. 1186/s12870-020-02621-y. 'Key Laboratory of Plant Resources Conservation and Sustainable Utilization / Guangdong Provincial Key Laboratory of Applied Botany, South China Botanical Garden, Chinese Academy of Sciences, Guangzhou, China. ${ }^{2}$ Center for Conservation Biology, Core Botanical Gardens, Chinese Academy of Sciences, Guangzhou, China. ${ }^{3}$ University of Chinese Academy of Sciences, Beijing, China. ${ }^{4}$ Independent Researcher, Guangzhou, China. ${ }^{5}$ Department of Genetics and Genome Biology, University of Leicester, Leicester LE1 7RH, UK. 
Received: 21 November 2019 Accepted: 25 August 2020

Published online: 02 September 2020

\section{References}

1. Qu XJ, Fan SJ, Wicke S, Yi YS. Plastome reduction in the only parasitic gymnosperm Parasitaxus is due to losses of photosynthesis but not housekeeping genes and apparently involves the secondary gain of a large inverted repeat. Genome Biol Evol. 2019;11(10):2789-96.

2. Daniell H, Lin CS, Yu M, Chang WJ. Chloroplast genomes: diversity, evolution, and applications in genetic engineering. Genome Biol. 2016;17: 134.

3. Moore MJ, Dhingra A, Soltis PS, Shaw R, Farmerie WG, Folta KM, et al. Rapid and accurate pyrosequencing of angiosperm plastid genomes. BMC Plant Biol. 2016;6:1-13.

4. Williams A, Miller JT, Small I, Nevill PG, Boykin LM. Integration of complete chloroplast genome sequences with small amplicon datasets improves phylogenetic resolution in Acacia. Mol Phylogenet Evol. 2016;96:1-8.

5. Shinozaki K, Ohme M, Tanaka M, Wakasugi T, Hayashida N, Matsubayashi T, et al. The complete nucleotide sequence of the tobacco chloroplast genome: its gene organization and expression. EMBO J. 1986;5(9):2043-9.

6. Tsunewaki K, Mori N, Takumi S. Experimental evolutionary studies on the genetic autonomy of the cytoplasmic genome "plasmon" in the Triticum (wheat)-Aegilops complex. Proc Natl Acad Sci U S A. 2019;116(8):3082-90.

7. Chiapella JO, Barfuss MHJ, Xue ZQ, Greimler J. The plastid genome of Deschampsia caespitosa (Poaceae). Molecules. 2019;24:216.

8. Yang JB, Tang M, Li HT, Zhang ZR, Li DZ. Complete chloroplast genome of the genus Cymbidium: lights into the species identification, phylogenetic implications and population genetic analyses. BMC Evol Biol. 2013;13:84.

9. Kim KJ, Choi KS, Jansen RK. Two chloroplast DNA inversions originated simultaneously during the early evolution of the sunflower family (Asteraceae). Mol Biol Evol. 2015;22(9):1783-92.

10. Katayama $\mathrm{H}$, Ogihara $\mathrm{Y}$. Phylogenetic affinities of the grasses to other monocots as revealed by molecular analysis of chloroplast DNA. Curr Genet. 1996;29(6):572-81.

11. Liu J, Qi ZC, Zhao YP, Fu CX, Xiang QY. Complete cpDNA genome sequence of Smilax china and phylogenetic placement of Liliales-influences of gene partitions and taxon sampling. Mol Phylogenet Evol. 2012;64(3): 545-62.

12. Salih RHM, Majeský L, Schwarzacher T, Gornall R, Heslop-Harrison P. Complete chloroplast genomes from apomictic Taraxacum (Asteraceae): identity and variation between three microspecies. PLoS One. 2017;12(2): e0168008.

13. Yi X, Gao L, Wang B, Su Y-J, Wang T. The complete chloroplast genome sequence of Cephalotaxus oliveri (Cephalotaxaceae): evolutionary comparison of Cephalotaxus chloroplast DNAs and insights into the loss of inverted repeat copies in gymnosperms. Genome Biol Evol. 2013;5(4):68898.

14. EFSA Panel on Dietetic Products and Nutrition and Allergies (EFSA). Scientific opinion on the substantiation of a health claim related to oat beta-glucan and lowering blood cholesterol and reduced risk of (coronary) heart disease pursuant to article 14 of regulation (EC) no 1924/2006. EFSA J. 2010;8(12):1885.

15. Loskutov IG, Rines HW. Avena L. In: Kole C, editor. Wild crop relatives: genomic and breeding resources, vol. 1. Heidelberg: Springer; 2011. p. 10984.

16. Liu Q, Lin L, Zhou XY, Peterson PM, Wen J. Unraveling the evolutionary dynamics of ancient and recent polyploidization events in Avena (Poaceae). Sci Rep. 2017;7:41944

17. Yan HH, Martin SL, Bekele WA, Latta RG, Diederichsen A, Peng YY, et al. Genome size variation in the genus Avena. Genome. 2016:59(3):209-20.

18. Luo MC, Gu YQ, Puiu D, Wang H. Genome sequence of the progenitor of the wheat D genome Aegilops tauschii. Nature. 2017;551(7681):498-502.

19. Fu YB. Oat evolution revealed in the maternal lineages of 25 Avena species. Sci Rep. 2018;8(1):4252.

20. Liu Q, Li XY, Zhou XY, Li MZ, Zhang FJ, Schwarzacher T, et al. The repetitive DNA landscape in Avena (Poaceae): chromosome and genome evolution defined by major repeat classes in whole-genome sequence reads. BMC Plant Biol. 2019;19(1):226.

21. Abdullah MF, Shahzadi I, Ali Z, Islam M, Naeem M, Mirza B, et al. Correlations among oligonucleotide repeats, nucleotide substitutions and insertion- deletion mutations in chloroplast genomes of plant family Malvaceae. J Syst Evol. 2020. https://doi.org/10.1111/jse.12585

22. Henriquez CL, Abdullah Al, Carlsen MM, Zuluaga A, Croat TB, McKain MR. Evolutionary dynamics in chloroplast genomes of subfamily Aroideae (Araceae). Genomics. 2020;112:2349-60.

23. Peng YY, Zhou PP, Zhao J, Li JZ, Lai SK, Tinker NA, et al. Phylogenetic relationships in the genus Avena based on the nuclear Pgk1 gene. PLoS One. 2018;13(11):e0200047.

24. Fu YB, Li P, Biligetu B. Developing chloroplast genomic resources from 25 Avena species for the characterization of oat wild relative germplasm. Plants. 2019:8(11):438.

25. Petkau A, Stuart-Edwards M, Stothard P, van Domselaar G. Interactive microbial genome visualization with GView. Bioinformatics. 2010;26(24): 3125-6.

26. Greiner S, Lehwark P, Bock R. OrganellarGenomeDRAW (OGDRAW) version 1 3.1: expanded toolkit for the graphical visualization of organellar genomes. Nucleic Acids Res. 2019;47(W1):W59-64.

27. Darling AE, Mau B, Perna NT. ProgressiveMauve: multiple genome alignment with gene gain, loss and rearrangement. PLoS One. 2010;5(6): e11147.

28. Frazer KA, Pachter L, Poliakov A, Rubin EM, Dubchak I. VISTA: computational tools for comparative genomics. Nucleic Acids Res. 2004;32(S2):W273-9.

29. Rozas J, Ferrer-Mata A, Sánchez-DelBarrio JC, Guirao-Rico S, Librado P, Ramos-Onsins SE, et al. DnaSP 6: DNA sequence polymorphism analysis of large datasets. Mol Biol Evol. 2017;34(12):3299-302.

30. Kurtz S, Choudhuri JV, Ohlebusch E, Schleiermacher C, Stoye J, Giegerich R. REPuter: the manifold applications of repeat analysis on a genomic scale. Nucleic Acids Res. 2001;29(22):4633-42.

31. Mayer C, Leese F, Tollrian R. Genome-wide analysis of tandem repeats in Daphnia pulex-a comparative approach. BMC Genomics. 2010;11(1):277.

32. Thiel T, Michalek W, Varshney R, Graner A. Exploring EST databases for the development and characterization of gene-derived SSR-markers in barley (Hordeum vulgare L.). Theor Appl Genet. 2003;106(3):411-22.

33. Dong WL, Wang RN, Zhang NY, Fan WB, Fang MF, Li ZH. Molecular evolution of chloroplast genomes of orchid species: insights into phylogenetic relationship and adaptive evolution. Int J Mol Sci. 2018;19(3):716.

34. Shermann-Broyles S, Bombarely A, Grimwood J, Schmutz J, Doyle J. Complete plastome sequences from Glycine syndetika and six additional perennial wild relatives of soybean. G3-genes. Genom Genet. 2014;4(10): 2023-33.

35. Gu ZG, Gu L, Eils R, Schlesner M, Brors B. circlize implements and enhances circular visualization in R. Bioinformatics. 2014;30(19):2811-2.

36. R Core Team. R: a language and environment for statistical computing. Vienna: R Foundation for Statistical Computing; 2020.

37. Akoglu H. User's guide to correlation coefficients. Turk J Emerg Med. 2018; 18(3):91-3.

38. Sharp PM, Li WH. Codon usage in regulatory genes in Escherichia coli does not reflect selection for rare codons. Nucleic Acids Res. 1986;14(19):7737-49.

39. Trifinopoulos J, Nguyen LT, von Haeseller A, Minh BQ. W-IQ-TREE: a fast online phylogenetic tool for maximum likelihood analysis. Nucleic Acids Res. 2016:44(W1):W232-5.

40. Guindon S, Dufayard JF, Lefort V, Anisimova M, Hordijk W, Gascuel O. New algorithms and methods to estimate maximum-likelihood phylogenies: assessing the performance of PhyML 3.0. Syst Biol. 2010;59(3):307-21.

41. Hoang DT, Chernomor O, von Haeseler A, Minh BQ, Le SV. UFBoot2: improving the ultrafast bootstrap approximation. Mol Biol Evol. 2017;35(2):518-22.

42. Darshetkar AM, Datar MN, Tamhankar S, Li P, Choudhary RK. Understanding evolution in Poales: insights from Eriocaulaceae plastome. PLoS One. 2019; 14(8):e0221423.

43. Huotari T, Korpelainen $\mathrm{H}$. Complete chloroplast genome sequence of Elodea canadensis and comparative analyses with other monocot plastid genomes. Gene. 2012;508(1):96-105.

44. Peredo EL, King UM, Les DH. The plastid genome of Najas flexilis: adaptation to submersed environments is accompanied by the complete loss of the $\mathrm{NDH}$ complex in an aquatic angiosperm. PLoS One. 2013;8(7):e68591.

45. Wicke S, Schneeweiss GM, de Pamphilis CW, Müller KF, Quandt D. The evolution of the plastid chromosome in land plants: gene content, gene order, gene function. Plant Mol Biol. 2011:76(3-5):273-97.

46. Peterson K, Schöttler MA, Karcher D, Thiele W, Bock R. Elimination of a group II intron from a plastid gene causes a mutant phenotype. Nucleic Acids Res. 2011;39(12):5181-92. 
47. Niu DK, Yang YF. Why eukaryotic cells use introns to enhance gene expression: splicing reduces transcription-associated mutagenesis by inhibiting topoisomerase I cutting activity. Biol Direct. 2011;6:24.

48. Emami S, Arumainayagam D, Korf I, Rose A. The effects of a stimulating intron of the expression of heterologous genes in Arabidopsis thaliana. Plant Biotechnol J. 2013;11(5):555-63.

49. Downie SR, Jansen RK. A comparative analysis of whole plastid genomes from the Apiales: expansion and contraction of the inverted repeat, mitochondrial to plastid transfer of DNA, and identification of highly divergent noncoding regions. Syst Bot. 2015;40(1):336-51.

50. Zhu A, Guo W, Gupta S, Fan W, Mower JP. Evolutionary dynamics of the plastid inverted repeat: the effects of expansion, contraction, and loss on substitution rates. New Phytol. 2016;209(4):1747-56.

51. Chumley TW, Palmer JD, Mower JP, Fourcade HM, Calie PJ, Boore JL, et al. The complete chloroplast genome sequence of Pelargonium $\times$ hortorum: organization and evolution of the largest and most highly rearranged chloroplast genome of land plants. Mol Biol Evol. 2006;23(11):2175-90

52. Lee HL, Jansen RK, Chumley TW, Kim KJ. Gene relocations within chloroplast genomes of Jasminum and Menodora (Oleaceae) are due to multiple, overlapping inversions. Mol Biol Evol. 2007;24(5):1161-80.

53. McDonald MJ, Wang WC, Huang HD, Leu JY. Clusters of nucleotide substitutions and insertion/deletion mutations are associated with repeat sequences. PLoS Biol. 2011;9(6):E1000622.

54. Mariotti R, Cultrera NG, Díez CM, Baldoni L, Rubini A. Identification of new polymorphic regions and differentiation of cultivated olives (Olea europaea L.) through plastome sequence comparison. BMC Plant Biol. 2010;10:211-23.

55. Wu DD, Sha LN, Tang C, Fan X, Wang Y, Kang HY, et al. The complete chloroplast genome sequence of Pseudoroegneria libanotica, genomic features, and phylogenetic relationship with Triticeae species. Biol Plantarum. 2018;62(2):231-40.

56. Qian J, Song JY, Gao HH, Zhu YJ, Xu J, Pang XH, et al. The complete chloroplast genome sequence of the medicinal plant Salvia miltiorrhiza. PLoS One. 2013;8(2):e57607.

57. Matsuoka Y, Yamazaki Y, Ogihara Y, Tsunewaki K. Whole chloroplast genome comparison of rice, maize, and wheat: implications for chloroplast gene diversification and phylogeny of cereals. Mol Biol Evol. 2002;19(12): 2084-91.

58. Wicke S, Müller KF, de Pamphilis CW, Quandt D, Bellot S, Schneeweiss GM. Mechanistic model of evolutionary rate variation en route to a nonphotosynthetic lifestyle in plants. Proc Natl Acad Sci U S A. 2016;113(32): 9045-50.

59. Abdullah MF, Shahzadi I, Waseem S, Mirza B, Ahmed I, Waheed MT. Chloroplast genome of Hibiscus rosa-sinensis (Malvaceae): comparative analyses and identification of mutational hotspots. Genomics. 2020;112(1): 581-91.

60. Li Y, Zhang ZR, Yang JB, Lv GH. Complete chloroplast genome of seven Fritillaria species, variable DNA markers identification and phylogenetic relationships within the genus. PLoS One. 2018;13(3):e0194613.

61. Iram S, Hayat MQ, Tahir M, Gul A, Abdullah Al. Chloroplast genome sequence of Artemisia scoparia: comparative analyses and screening of mutational hotspots. Plants. 2019;8(11):476.

62. Li LD, Jiang Y, Liu YY, Niu ZT, Xue QY, Liu W, et al. The large single-copy (LSC) region functions as a highly effective and efficient molecular marker for accurate authentication of medicinal Dendrobium species. Acta Pharm Sin B. 2020. https://doi.org/10.1016/j.apsb.2020.01.012.

63. Henriquez CL, Abdullah Al, Carlsen MM, Zuluaga A, Croat TB, Mckain MR. Molecular evolution of chloroplast genomes in Monsteroideae (Araceae). Planta. 2020;1:72.

64. Dierckxsens N, Mardulyn P, Smits G. NOVOPlasty: de novo assembly of organelle genomes from whole genome data. Nucleic Acids Res. 2017;45(4):e18.

65. Kearse M, Moir R, Wilson A, Stones-Havas S, Cheung M, Sturrock S, et al. Geneious basic: an integrated and extendable desktop software platform for the organization and analysis of sequence data. Bioinformatics. 2012;28(12):1647-9.

66. Luo RB, Liu BH, Xie YL, Li ZY, Hunag WH, Yuan JY, et al. SOAPdenovo2: an empirically improved memory-efficient short-read de novo assembler. Gigascience. 2012;1:18

67. Zerbino DR, Birney E. Velvet: algorithms for de novo short read assembly using de Bruijn graphs. Genome Res. 2008;18(5):821-9.

68. Tillich M, Lehwark P, Pellizzer T, Ulbricht-Jones ES, Fischer A, Bock R, et al. GeSeq- versatile and accurate annotation of organelle genomes. Nucleic Acids Res. 2017:45(W1):W6-W11.
69. Warburton PE, Giordano J, Cheung F, Gelfand Y, Benson G. Inverted repeat structure of the human genome: the X-chromosome contains a preponderance of large, highly homologous inverted repeats that contain testes genes. Genome Res. 2004;14(10A):1861-9.

70. Brudno M, Malde S, Poliakov A, Do CB, Couronne O, Dubchak I, et al. Glocal alignment: finding rearrangements during alignment. Bioinformatics. 2003; 19(suppl. 1):i54-62.

71. Amiryousefi A, Hyvönen J, Poczai P. IRscope: an online program to visualize the junction sites of chloroplast genomes. Bioinformatics. 2018;34(17):3030 1.

72. Wang D, Zhang Y, Zhang Z, Zhu J, Yu J. KaKs_Calculator 2.0: a toolkit incorporating gamma-series methods and sliding window strategies. Genomics Proteomics Bioinformatics. 2010;8(1):77-80.

73. Soreng RJ, Peterson PM, Romaschenko K, Davidse G, Teisher JK, Clark LG, et al. A worldwide phylogenetic classification of the Poaceae (Gramineae) II: an update and a comparison of two 2015 classifications. J Syst Evol. 2017; 55(4):259-90.

74. Hall TA. BioEdit: a user-friendly biological sequence alignment editor and analysis program for windows 95/98/nt. Nucleic Acids Symp Ser. 1999;41(1): 95-8.

75. Darriba D, Taboada GL, Doallo R, Posada D. jModelTest 2: more models, new heuristics and parallel computing. Nat Methods. 2012;9(8):772.

76. Tamura K, Stecher G, Peterson D, Filipski A, Kumar S. MEGA6: molecular evolutionary genetics analysis version 6.0. Mol Biol Evol. 2013;30(12):2725-9.

\section{Publisher's Note}

Springer Nature remains neutral with regard to jurisdictional claims in published maps and institutional affiliations.

\section{Ready to submit your research? Choose BMC and benefit from:}

- fast, convenient online submission

- thorough peer review by experienced researchers in your field

- rapid publication on acceptance

- support for research data, including large and complex data types

- gold Open Access which fosters wider collaboration and increased citations

- maximum visibility for your research: over $100 \mathrm{M}$ website views per year

At BMC, research is always in progress.

Learn more biomedcentral.com/submissions 